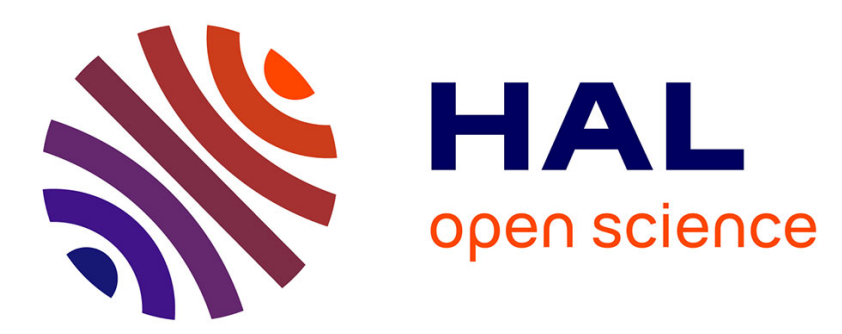

\title{
Linear and ultrafast nonlinear plasmonics of single nano-objects
}

\author{
Aurélien Crut, Paolo Maioli, Fabrice Vallée, Natalia del Fatti
}

\section{To cite this version:}

Aurélien Crut, Paolo Maioli, Fabrice Vallée, Natalia del Fatti. Linear and ultrafast nonlinear plasmonics of single nano-objects. Journal of Physics: Condensed Matter, 2017, 29 (12), 10.1088/1361$648 \mathrm{X} / \mathrm{aa} 59 \mathrm{cc}$. hal-01698280

\section{HAL Id: hal-01698280 \\ https://univ-lyon1.hal.science/hal-01698280}

Submitted on 1 Feb 2018

HAL is a multi-disciplinary open access archive for the deposit and dissemination of scientific research documents, whether they are published or not. The documents may come from teaching and research institutions in France or abroad, or from public or private research centers.
L'archive ouverte pluridisciplinaire HAL, est destinée au dépôt et à la diffusion de documents scientifiques de niveau recherche, publiés ou non, émanant des établissements d'enseignement et de recherche français ou étrangers, des laboratoires publics ou privés. 


\title{
Linear and ultrafast nonlinear plasmonics of single nano-objects
}

\author{
Aurélien Crut, Paolo Maioli, Fabrice Vallée and Natalia Del Fatti \\ FemtoNanoOptics group, Institut Lumière Matière UMR5306, Université Lyon 1, CNRS, \\ Université de Lyon, 69622 Villeurbanne, France \\ *Corresponding author: natalia.del-fatti@univ-lyon1.fr
}

\begin{abstract}
\end{abstract}
Single-particle optical investigations have greatly improved our understanding of the fundamental properties of nano-objects, avoiding the spurious inhomogeneous effects that affect ensemble experiments. Correlation with high-resolution imaging techniques providing morphological information (e.g., electron microscopy) allows a quantitative interpretation of the optical measurements by means of analytical models and numerical simulations. In this topical review, we first briefly recall the principles underlying some of the most commonly used single-particle optical techniques: near-field, dark-field, spatial modulation and photothermal microscopies/spectroscopies. We then focus on the quantitative investigation of the surface plasmon resonance (SPR) of metallic nano-objects using linear and ultrafast optical techniques. While measured SPR positions and spectral areas are found in good agreement with predictions based on Maxwell's equations, SPR widths are strongly influenced by quantum confinement (or, from a classical standpoint, surface-induced electron scattering) and, for small nano-objects, cannot be reproduced using the dielectric functions of bulk materials. Linear measurements on single nanoobjects (silver nanospheres and gold nanorods) allow a quantification of the size and geometry dependences of these effects in confined metals. Addressing the ultrafast response of an individual nano-object is also a powerful tool to elucidate the physical mechanisms at the origin of their optical nonlinearities, and their electronic, vibrational 
and thermal relaxation processes. Experimental investigations of the dynamical response of gold nanorods are shown to be quantitatively modeled in terms of modifications of the metal dielectric function enhanced by plasmonic effects. Ultrafast spectroscopy can also be exploited to unveil hidden physical properties of more complex nanosystems. In this context, two-color femtosecond pump-probe experiments performed on individual bimetallic heterodimers are discussed in the last part of the review, demonstrating the existence of Fano interferences in the optical absorption of a gold nanoparticle under the influence of a nearby silver one.

\section{Introduction}

Understanding and modeling the optical linear and nonlinear properties of nanoobjects have led to considerable activities in the academic and industrial domains during the last two decades. This large interest is motivated by the modification of the optical properties of a material with size reduction, and their concomitant dependencies on the morphology, composition, and environment of a nano-object. These dependencies open up many possibilities for their control and design, provided their physical origins are precisely understood and modeled, and thus for many applications such as optical sources, sensors and detectors. Though much information has been obtained by investigating ensembles of nanoparticles, the results of such experiments are affected by averaging effects due to unavoidable dispersions of the particle morphology and environment characteristics. These issues limit precise comparison with theoretical models of key features of the optical response of nano-object, such as their optical resonance width $[1,2]$, or the investigation of specific nano-objects synthesized with a relatively low percentage yield [3]. 
This has fostered the development of novel experimental methods to optically detect and investigate individual nano-objects, which has represented an important step forward in nanosciences and nanotechnology. From a fundamental point of view, these methods make possible a precise correlation of the optical properties of a nano-object (absorption, scattering, luminescence, ultrafast optical response,...) with its internal (composition, morphology, crystalline structure) and external (solid or liquid environment, substrate, molecules bound at its surface, interactions with other nano-objects,...) characteristics, provided these can be determined for the studied particle. Such a correlation yields information for optimizing the optical response of nano-objects, designing new ones, and addressing a single one to create ultimate optical nanodevices such as emitters [4], biomarkers [5] or high-sensitivity sensors [6]. Furthermore, this also opens the way to fundamental investigation of the physical properties of a single nano-object using linear or nonlinear optical spectroscopies, e.g., electron interactions and kinetics, lattice vibrations, thermal and mechanical energy exchanges or magnetism, in particular using time-resolved techniques [7-10].

Methods for optical detection and investigation of an individual nano-object have been developed using different aspects of its interaction with light such as elastic (Rayleigh) and inelastic (Brillouin, Raman) scattering, absorption, photoluminescence, second- or thirdharmonic generation, four-wave mixing. For highly luminescent nano-objects (e.g., quantum dots and semiconductor carbon nanotubes), high sensitivity detection of the emitted luminescence light after its spectral filtering makes their optical observation quasi-routine experiments $[11,12]$. This approach has been exploited in a large variety of applications ranging from quantum optics studies [13] to biological labeling [5]. Development of nonluminescence based methods has been achieved during the last decade, either detecting 
absorption or scattering of light incident on a nano-object using various high sensitivity approaches (e.g., dark-field microscopy [14,15], interferometry [16], spatial modulation $[17,18]$, and Raman spectroscopy $[19,20])$ or exploiting nonlinear optical methods (photothermal [21-23] and transient absorption [24] imaging, second- and third-harmonic generation [25,26], and four-wave mixing [27]). They have opened the way to detailed optical investigations of single weakly luminescent nano-objects, such as metallic or dielectric nanoparticles, or metallic carbon nanotubes, down to few nanometer sizes $[17,22,28]$ (note that some of these nano-objects can be detected by photoluminescence despite its poor quantum efficiency [29]). They have also provided additional information (e.g., direct measurement of the absorption or scattering spectra) on luminescent nanosystems such as quantum dots [30], semiconductor nanowires [31], semiconductor carbon nanotubes [28,32], and molecules [33].

In the case of single metal nanoparticles, the first studies were performed using nearfield optical techniques [34]. Though they offer the advantage of improved spatial resolution (circumventing the Rayleigh-Abbe limit) and ability to image electromagnetic field distributions [35], they are experimentally challenging and require complex analysis of experimental data to take into account modification of the particle response due to the presence of a tip in its near-field region. Investigation of the linear scattering, extinction or absorption spectra of metal nanoparticles are thus mostly performed with far-field techniques, i.e., using light sources and detectors located at a distance from the particle much larger than light wavelength. The most commonly used approaches are dark-field microscopy, spatial modulation spectroscopy, and photothermal imaging (figure 1). The performed studies have considerably improved our understanding of the optical properties of metal nanoparticles, and, in particular, of the characteristics of their localized Surface 
Plasmon Resonance (SPR) [36,37], the key feature for their optical applications. For instance, their sensitivity to the particle environment has been exploited for biosensing $[6,38]$, and the local electromagnetic field enhancement associated to the SPR for enhancing the Raman or fluorescence responses of surrounding molecules $[39,40]$. Optimizing the efficiency of such applications requires adjusting the SPR wavelength to the desired spectral range and enhancing the SPR effect, i.e., increasing its peak amplitude and reducing its spectral width, both requiring precise understanding and modeling of the SPR characteristics as a function of the particle composition, morphology and environment.

Many results have now been obtained on single nanoparticles of different size, shape, composition, and environment, and precisely compared to theoretical models provided their morphology could be determined using characterization tools such as electron microscopy (using SEM or TEM) [41,42]. In this context, spatial modulation spectroscopy (SMS $[17,18])$ is particularly interesting as it yields the quantitative extinction spectrum of a nano-object (i.e., not only the spectral dispersion of its extinction crosssection, but also its absolute amplitude). In section 2, after briefly reviewing single-particle optical techniques, mostly focusing on the SMS one, we will describe their application to experimental determination of the SPR characteristics (amplitude, spectral position and width) of a metal nanoparticle, and the comparison of the experimental results to those of classical electrodynamics calculations. Special emphasis will be given to experimental determination of the SPR width of relatively small size nano-objects (below typically 20-30 $\mathrm{nm}$ ), as it is significantly modified by electron quantum confinement, a contribution still little quantitatively modeled $[1,43]$. By combining SMS with a femtosecond pump-probe setup, ultrafast investigations can also be performed on the same linearly characterized nanoobject, yielding access to fundamental physical properties such as the origin of optical 
nonlinearities in confined metals, or the existence of Fano effect in the absorption of plasmonic nanoparticles in interaction with each other [44-46]. This will be discussed in section 3.

\section{Plasmonic response of a single metal nano-object}

\subsection{Single particle optical investigations}

The different linear optical methods for investigation of a single nanoparticle are based on detection of its light scattering, absorption or extinction. The measured optical signals are thus connected to the absorption, $\sigma_{a b s}$, scattering, $\sigma_{s c a t}$, or extinction, $\sigma_{e x t}=\sigma_{a b s}+\sigma_{s c a t}$, crosssections that globally describe the response of the investigated particle in its environment. The amplitude and dispersion of these cross-sections reflect the spatial distribution of the electromagnetic field in and around the particle. Outside the particle, the total electric field $\boldsymbol{E}$ can be separated into the incident field $\boldsymbol{E}_{\boldsymbol{i}}$, solution of Maxwell's equations in its absence (including refraction/reflection at interfaces for inhomogeneous environment, e.g., for a substrate-deposited particle), and the electric field $\boldsymbol{E}_{\text {scat }}$ that it scatters:

$$
E=E_{i}+E_{\text {scat }}
$$

$\boldsymbol{E}_{\text {scat }}$ includes evanescent waves in the near-field region (distances smaller than the wavelength $\lambda$ of the incident light beam) and reduces to propagating waves in the far-field one (distances $>>\lambda$ ). Describing the particle and the different elements of its environment (matrix, substrate) by their dielectric functions, $\boldsymbol{E}_{\text {scat }}$, together with the field in the particle, can be (numerically, except for systems with high symmetry) computed by solving Maxwell's equations with the proper boundary conditions. The scattered field fully reflects the optical 
response of the particle, as its scattering cross-section $\sigma_{\text {scat }}$ is related to the total scattered energy (proportional in far field to $\iint_{S}\left\langle\left|\boldsymbol{E}_{\text {scat }}\right|^{2}\right\rangle_{t} d S$, where $S$ is a spherical surface surrounding the particle and \langle\rangle$_{t}$ stands for time averaging), while $\sigma_{\text {ext }}$ can be deduced from the forward scattering amplitude for plane wave illumination (optical theorem) $[8,47]$.

In a single-particle experiment, light energy is collected by a photodetector over a finite solid angle $\Omega$ (defining a portion $S_{\Omega}$ of the previous spherical surface), yielding a signal proportional to $\iint_{S_{\Omega}}\left\langle|\boldsymbol{E}|^{2}\right\rangle_{t} d S_{\Omega}$, where $\boldsymbol{E}$ is given by equation 1 (the same expression can be extended to interferometric approaches using a reference beam propagating away from the particle, replacing $\boldsymbol{E}$ by $\boldsymbol{E}+\boldsymbol{E}_{\text {ref }}, \boldsymbol{E}_{\text {ref }}$ being the reference electric field $\left.[16,21,48]\right)$. The optical signature of the particle, i.e., its scattered field, is thus detected via its interference with the incident field $\boldsymbol{E}_{\boldsymbol{i}}$, in a configuration depending on the considered optical setup (figure 1). The measured signal thus a priori contains pure scattering $\left(\left\langle\left|E_{\text {scat }}\right|^{2}\right\rangle_{\mathrm{t}}\right.$ term) and extinction $\left(\left\langle E_{\text {scat }} E_{i}\right\rangle_{\mathrm{t}}\right.$ term $)$ contributions $[8,47,49]$, with relative weights depending on the experimental geometry and nanoparticle morphology (due to the different sizedependencies of the scattering and absorption cross-sections) [49].

The spatial resolution of the far-field techniques, typically half of the wavelength, requires use of diluted samples, with surface density of typically one particle per $\mu \mathrm{m}^{2}$. Darkfield microscopy, the most largely used optical technique for individual nanoparticle studies, is based on illuminating the particle using a hollow light cone with an internal angle exceeding the angular aperture of the microscope objective collecting the scattered light (figure 1a) $[14,50]$. Alternatively, an illumination scheme based on total internal reflection can also be used [51,52]. In both cases, the scattered light is selectively detected, with a 
measured signal proportional to $\iint_{S_{\Omega}}\left\langle\left|\boldsymbol{E}_{\text {scat }}\right|^{2}\right\rangle_{t} d S_{\Omega}$, and thus to the scattering cross section, $\sigma_{\text {scat }}$, of the particle. As dark-field microscopy relies on detection of light scattering, the detected signal quickly decreases with particle size reduction $\left(\sigma_{\text {scat }}\right.$ decreases proportionally to the square of the particle volume, $V^{2}$, for sizes much smaller than $\lambda$ ). It is thus limited to relatively large nanoparticles, typically larger than $20 \mathrm{~nm}$ [53]. This size range is of large interest for many synthesized systems and, because of its simplicity and ease of operation, dark-field microscopy has been extensively used for single particle spectroscopy. Extension to smaller sizes, down to less than $10 \mathrm{~nm}$, has been performed using a heterodyne method, however making the approach more complex [54]. It has also been extended to wide-field imaging mode, permitting tracking of an individual particle or simultaneous monitoring of a large number of them [55]. The major limitation of dark-field microscopy is the difficulty of determining the absolute value of the particle scattering cross-section, as the measured signal depends on the fraction of the collected scattered light, and thus on the scattering pattern of the object [56]. Addressing single metal nanoparticles of small sizes is more readily done measuring a signal associated to the particle absorption, the latter strongly dominating the optical response of small particles $\left(\sigma_{a b s}\right.$ is about equal to $\sigma_{\text {scat }}$ for a $80 \mathrm{~nm}$ diameter gold nanosphere, and 100 times larger for a $20 \mathrm{~nm}$ one). Absorption-based techniques require detection of the incident light beam (e.g., of the transmitted or reflected wave in the most usual case of substrate-deposited nano-objects), leading to a signal proportional to $\left\langle\left|E_{i}+E_{\text {scat }}\right|^{2}\right\rangle_{\mathrm{t}}$, with for small nano-objects a scattered field amplitude much smaller than the incident one, $E_{\text {scat }} \ll<E_{\mathrm{i}}$. Detecting the presence of such a small nano-object thus requires the development of high sensitivity detection schemes, using differential detection [33] or field modulations. The latter approaches, including photothermal 
spectroscopy [22] and spatial modulation spectroscopy [17], are based on time modulation of $\boldsymbol{E}_{\text {scat }}$ and synchronous detection of the measured signals, permitting observation of particles with sizes down to a few $\mathrm{nm}$.

In photothermal spectroscopy, modulation is performed using a time-modulated pump beam that heats-up the nanoparticle and, by heat transfer, its environment, assumed non-absorbing at the pump beam wavelength. This induces changes of the refractive index in and around the nanoparticle, which reflect in modulation of the scattering of a second probe beam (figure 1d). This nonlinear optical technique (the optical properties of a light beam are modulated by another one) yields a signal proportional to the nanoparticle absorption. This approach was first demonstrated in reflection, with interferometric detection of the reflected probe beam using a third reference beam (photothermal interference contrast, PIC) [21]. Direct detection of the probe beam in reflection or transmission, without reference, was further performed (photothermal heterodyne imaging, PHI, figure 1d). This simpler and more sensitive experimental scheme permits detection of gold clusters as small as $1.4 \mathrm{~nm}$ [22], and was further extended to other absorbing nanoobjects, such as semiconductor quantum dots and carbon nanotubes [22,57], and to single molecules [58]. As it is insensitive to light scattering, this method is well adapted to biological imaging due to its ability to detect metal nanoparticles in highly scattering environments, as demonstrated for $5 \mathrm{~nm}$ gold nanoparticles in live neurons [59]. Apart from its experimental complexity, involving at least two overlapping light beams, the major limitation of photothermal techniques is the difficulty to connect the measured signal amplitude to the absorption cross-section of a nanoparticle (knowledge of thermal dynamics being in particular required) [60]. 
Quantitative measurements of the extinction cross-section of a nanoparticle can be performed by spatial modulation spectroscopy, based on modulation of $\boldsymbol{E}_{\text {scat }}$ via modulation of the particle position under a tightly focused light beam (Figure 1b). In transmission SMS (T-SMS), the transmitted light is collected through a second microscope objective, with the same numerical aperture as the focusing one, detecting the transmitted power $P_{t}=2 c \varepsilon_{0} \iint_{S_{\Omega}}\left\langle\left|E_{i}+E_{\text {scat }}\right|^{2}\right\rangle_{t} d S_{\Omega}$. Assuming the particle much smaller than the focal spot size $d$ at position $\left(x_{0}, y_{0}\right)$ in the focal plane, and neglecting substrate effects (discussed in [8]) for the sake of simplicity, $P_{t}$ is given by $[8,47]$ :

$$
P_{t}=P_{i}-\sigma_{e x t} I\left(x_{0}, y_{0}\right)+2 c \varepsilon_{0} \iint_{S_{\Omega}}\left\langle\left|\boldsymbol{E}_{\text {scat }}\right|^{2}\right\rangle_{t} d S_{\Omega} \approx P_{i}-\sigma_{e x t} I\left(x_{0}, y_{0}\right)
$$

where $P_{i}$ is the incident power and $I(x, y)$ the intensity profile of the illuminating beam at its focal plane. The transmission change $P_{i}-P_{t}$ is thus directly proportional to the extinction cross-section $\sigma_{\text {ext, }}$ provided the collected scattered light can be neglected (e.g., for sufficiently small objects for which $\sigma_{\text {scat }} \ll \sigma_{\text {ext }}$, and/or not too large collecting solid angle, $\Omega$ ). Modulating the particle position at frequency $f$ (typically $1.5 \mathrm{kHz}$ ) along the $y$ direction, $x=x_{0}$ and $y=y_{0}+\delta \sin (2 \pi f t)$, with an amplitude $\delta$ much smaller than $d$, and developing $P_{t}$ up to the second order in $\delta$ one gets:

$$
P_{i}-P_{t} \approx \sigma_{e x t} I\left(x_{0}, y_{0}\right)+\sigma_{e x t} \frac{\partial I}{\partial y}\left(x_{0}, y_{0}\right) \delta \sin (2 \pi f t)+\frac{1}{4} \sigma_{e x t} \frac{\partial^{2} I}{\partial y^{2}}\left(x_{0}, y_{0}\right) \delta^{2}[1-\cos (4 \pi f t)]
$$


Selecting the $f$ or $2 f$ modulated part of $P_{t}$ with a lock-in amplifier, and scanning the sample position in the focal plane (i.e., changing $x_{0}$ and $y_{0}$ ), a particle thus shows up as a signal proportional to the first or second derivative of $I$ in the $y$ direction (figure 1c). Similar shapes are numerically computed for larger $\delta$ values, yielding optimal modulation amplitude of the order of the spot size $d$ [61]. Using this approach, gold and silver nanoparticles as small as 5 and $2 \mathrm{~nm}$ can be detected [17]. Furthermore, as the $f$ or $2 f$ modulated part of $P_{t}$ (or equivalently of the relative transmission change $\left.\Delta T / T=\left(P_{t^{-}}-P_{i}\right) / P_{i}\right)$ is directly proportional to $\sigma_{\text {ext, }}$ with a proportionality factor fully determined by two measurable experimental parameters, $\delta$ and $d$, the $\sigma_{\text {ext }}$ amplitude can be determined. Associated to spectrally dependent measurements, using a wavelength tunable light source (tunable laser $[3,62]$, supercontinuum source $[61,63]$ or white lamp [64]) and light polarization dependent studies $[65,66]$, the full characteristics (amplitude, spectrum and polarization dependence) of the extinction cross section of a single nanoparticle can be determined. First applied to metal nanoparticles of different shapes and environment $[8,17,65]$, SMS was further extended to other systems such as carbon nanotubes and semiconductor quantum dots and nanowires $[18,32,67,68]$.

SMS has been further extended to reflection (R-SMS), making it applicable to nanoobjects deposited on an opaque substrate, a situation frequently encountered when using top-down nanofabrication techniques (figure 1b). It was first applied to measurement of the absorption of individual single wall carbon nanotubes on a silica-on-silicon substrate (figure 1c) [18] and, combining T-SMS and R-SMS, to compare the absorption spectra of the suspended or deposited sections of the same carbon nanotube [32]. As for T-SMS, the amplitude of the measured relative reflectivity change $\Delta R / R$ is connected to $\sigma_{\text {ext }}$, permitting 
its quantitative determination, provided Fabry-Perot effects are taken into account when layered substrates are used [18].

When investigating the properties of a single nano-object, the precise determination of its morphology is a key issue, especially for comparing experimental and theoretical results. In the context of optical studies, large efforts have thus been devoted to perform optical measurements and morphology characterization on the same nano-object. This is most usually done using either atomic force microscopy [18] or transmission or scanning electron microscopies (TEM or SEM). In the latter cases, adapted substrates have to be used, i.e., thin ones transparent to both photons and electrons (e.g., a $40 \mathrm{~nm}$ silica one) [41,69-71] or conducting ones (e.g., ITO) [50,72], respectively. However, as electron exposure may affect the object properties, and in particular its optical spectra, care must be taken performing optical and electron microscopy imaging studies on the same nano-object. It has been shown that individual nano-object imaging with low magnification and electron fluence does not modify their optical response $[3,62]$, and permits their crude identification prior to their optical study and further higher-magnification electron microscopy imaging [3]. The combination of the optical spectroscopy of a single nanoparticle with its independent morphology characterization has opened the way to a precise and quantitative analysis of the impact of size, shape and environment effects on the optical response of metal nanoparticles, and in particular of their surface plasmon resonance.

\subsection{Surface plasmon resonance of metal nano-objects}

Metal nanoparticles support localized surface plasmon resonances, which strongly modify their optical response as compared to bulk metals. From a classical point of view, these resonances result from collective electron oscillations induced by the external electromagnetic field. The electronic movement creates an electromagnetic field which 
adds-up to the initial one, leading to strong spatial variation and enhancement of the full field in and in vicinity of the particle around specific wavelengths. This reflects in enhancements of the particle optical scattering and absorption cross-sections, which show as resonances in their spectra. Their spectral characteristics, i.e., peak wavelength, spectral shape, and amplitude, depend on the nanoparticle composition, morphology and environment [37].

As SPR is a classical electromagnetic effect, its spectral characteristics can be modeled using a method describing the nanoparticle and the different elements of its surrounding (substrate, matrix, coating layer, other nanoparticles, ...) by their respective dielectric functions (with quantum modification when small sizes or separating distances are involved $[43,66,73,74])$. The problem then reduces to solving Maxwell's equations with proper electromagnetic field boundary conditions at interfaces. It can be solved analytically for a sphere or an infinite cylinder in a homogeneous matrix (using a multipolar expansion of the electromagnetic field, Mie theory) and numerically for arbitrary nano-object shape and environment, using numerical approaches such as discrete-dipole approximation (DDA), finite difference time domain (FDTD), boundary element method (BEM) or finite element method (FEM).

Apart from knowledge of the full geometry of the particle and environment, the key parameters in the modeling are thus the dielectric functions of the involved materials. For a noble metal nanoparticle, the confined metal dielectric function can be separated into the sum of interband, $\varepsilon^{i b}$, and intraband (Drude-like) components: 


$$
\varepsilon(\omega)=\varepsilon^{i b}-\frac{\omega_{p}^{2}}{\omega(\omega+i \gamma)} \approx \varepsilon_{1}^{i b}-\frac{\omega_{p}^{2}}{\omega^{2}}+i\left[\varepsilon_{2}^{i b}+\frac{\omega_{p}^{2} \gamma}{\omega^{3}}\right]
$$

where $\omega_{p}$ is the plasma frequency of the metal and $\gamma$ the optical scattering rate of conduction electrons in the particle (with $\gamma \ll \omega$ ). The $\varepsilon^{b}$ term, associated to interband transitions (figure 2), is not significantly modified for sizes above about 2-3 nm [1]. Size reduction mostly reflects in a size dependence of $\gamma$ due to additional electron scattering by the surface of the particle when its size becomes smaller than the bulk electron mean free path [75]. $\gamma$ can thus be written as the sum $\gamma=\gamma_{0}+\gamma_{s}$ of the bulk-like optical scattering rate $\gamma_{0}$ (due to electron-phonon and umklapp electron-electron scattering) and of a size-dependent term $\gamma_{s}$, classically interpreted as electron scattering at nanoparticle surface.

For a particle much smaller than the optical wavelength, the incident electromagnetic field can be assumed uniform over its size, leading to a purely dipolar particle response (figure 2a). The extinction cross-section of a nanoellipsoid in this regime, which can be used as a starting point to understand the optical properties of both nanospheres and elongated nano-objects, is:

$$
\sigma_{e x t}^{i}(\omega) \approx \sigma_{a b s}^{i}(\omega)=\frac{\omega V \varepsilon_{m}{ }^{3 / 2}}{c} \frac{\varepsilon_{2}(\omega)}{\left[L_{i} \varepsilon_{1}(\omega)+\left(1-L_{i}\right) \varepsilon_{m}\right]^{2}+\varepsilon_{2}{ }^{2}(\omega)}
$$

where $V$ is the nanoparticle volume, $\omega$ the incident light frequency, $c$ the light velocity in vacuum and $\varepsilon=\varepsilon_{1}+\mathrm{i} \varepsilon_{2}$ and $\varepsilon_{m}$ are the dielectric functions of the nanoellipsoid and of its environment (the latter assumed to be real and undispersed, absorbing environments being discussed in [76]), respectively. The $L_{i}$ are shape-dependent factors characterizing the optical 
response for light polarized along the $i$ axis of the nanoellipsoids $[47,77]$, with $L_{i}=1 / 3$ for nanospheres. If $\varepsilon^{i b}$ is small or weakly dispersed close to the SPR frequency (as for noble metals away from interband transitions, e.g. for silver nanospheres or elongated gold nanoobjects), the $\sigma_{\text {ext }}$ spectrum (equation 5 ) displays a quasi-Lorentzian profile in the energy domain, thus described by three independent parameters: spectral position $\left(\Omega_{R}\right)$, area $(\Xi)$ and width $(\Gamma)$

$$
\sigma_{e x t}(\omega)=\Xi \frac{\Gamma /(2 \pi)}{\left(\omega-\Omega_{R}\right)^{2}+(\Gamma / 2)^{2}}
$$

The central frequency $\Omega_{R}$ of the resonance is given by the condition $L_{i} \varepsilon_{1}\left(\Omega_{R}\right)+\left(1-L_{i}\right) \varepsilon_{m}=0$, its area by $\Xi=\pi V \varepsilon_{m}{ }^{3 / 2} \Omega_{R}{ }^{4} /\left(2 c L_{i}^{2} \omega_{p}{ }^{2}\right)$ and its non-radiative width $\Gamma_{n r}$ by:

$$
\Gamma_{n r}=\frac{\varepsilon_{2}\left(\Omega_{R}\right) \Omega_{R}{ }^{3}}{\omega_{p}^{2}}=\gamma+\frac{\varepsilon_{2}{ }^{i b}\left(\Omega_{R}\right) \Omega_{R}{ }^{3}}{\omega_{p}^{2}}
$$

In the general case (i.e., for a nanoparticle of arbitrary size), both radiative and nonradiative processes contribute to the damping rate $\Gamma$ (figure 2a). Radiative damping reflects surface plasmon relaxation by coupling with light (i.e., reemission of photons by Rayleigh scattering, with an associated rate $\Gamma_{\mathrm{r}}$ ), with an efficiency increasing with nanoparticle size. Nonradiative Landau damping (associated rate $\Gamma_{n r}$ ) represents surface plasmon decay into single electronic excitations (absorption), associated to either an intraband transition within the conduction band (first term in the right part of equation 7), or an interband transition implying a departure state in the valence band (second term in the right part of equation 7). 
While well-understood for bulk materials, description of Landau damping in small nanoparticles is complex due to the size-dependent variations of $\gamma$ (through the $\gamma_{s}$ term). Importantly, this contribution affects the imaginary part of the dielectric function $\left(\varepsilon_{2}\right)$, and thus the SPR width $\Gamma$ (equation 7) but not SPR position $\left(\Omega_{R}\right)$ and area $(\Xi)$ which are related to $\varepsilon_{1}$. The total damping rate $\Gamma$ thus writes as:

$$
\Gamma\left(\Omega_{R}\right)=\Gamma_{r}\left(\Omega_{R}\right)+\Gamma_{n r}\left(\Omega_{R}\right)=\Gamma_{r}\left(\Omega_{R}\right)+\Gamma_{0}\left(\Omega_{R}\right)+\gamma_{s}
$$

where $\Gamma_{0}=\Gamma_{i b}+\gamma_{0}$ is the bulk-like non-radiative broadening term, including both interband ( $\left.\Gamma_{i b}=\frac{\varepsilon_{2}{ }^{i b}\left(\Omega_{R}\right) \Omega_{R}{ }^{3}}{\omega_{p}^{2}}\right)$ and intraband contributions, and $\gamma_{s}$ reflects the size-dependent contribution due to electron quantum confinement in the nanoparticle (its weak frequency dependence being neglected here).

\subsection{Investigating single nanoparticle SPR spectral position and area: size and environment effects}

According to equations 5-7, SPR position $\Omega_{R}$ and area $\Xi$ are related to nano-object shape, volume and environment refractive index and are weakly affected by quantum effects in the low size regime. For small nanospheres (with a diameter $<20 \mathrm{~nm}$ ), $\Omega_{R}$ is predominantly determined by the particle's environment (the resonance condition reducing to $\varepsilon_{1}+2 \varepsilon_{m}=0$ ), while $\Xi$ depends on both the particle volume and the environment's refractive index. Therefore the knowledge of the $\sigma_{\text {ext }}$ spectrum of a nanosphere allows determination of both its diameter and environment refractive index, using equation 5 or Mie theory. This analysis can be extended to slightly elongated nanoparticles, by using the polarization dependence of $\sigma_{\text {ext }}$ spectra $[41,61,66]$. The sizes optically deduced from a quantitative 
reproduction of the SMS spectra (presented in more detail in Figure 5 and below) have been demonstrated to coincide with those directly determined by electron microscopy, as shown by comparison on statistical distributions [61] or at the single-particle level [41].

For more complex morphologies, such as nanorods and nanobipyramids, the dependence of the relationship between $\sigma_{\text {ext }}$ spectra and the characteristics of a particle and its environment cannot be easily disentangled, as $\Omega_{R}$ and $\Xi$ depend on both the aspect ratio and environment of nano-objects. This makes it difficult to fully characterize the particle geometry from optical measurements alone. It is however possible to optically determine the dimensions of a simple nano-object provided its environment is well characterized (e.g., if it is embedded or encapsulated in a dielectric medium of known refractive index), or to access the environment properties of a nano-object whose dimensions have been determined by electron microscopy. An example of the former analysis is demonstrated in figure 3. In this situation, the local environment of a gold nanorod was controlled by its silica encapsulation (figure 3a), so that SPR properties are weakly sensitive to the external fluctuating environment of the $\mathrm{Au}_{\mathrm{SiO}}$ nanorod [69]. An optimal reproduction of the measured $\Omega_{R}$ and $\Xi$ in the frequency domain (figure $3 \mathrm{~b}$ ) and $\sigma_{\text {ext }}$ polarization dependence (figure 3c) were obtained in FEM calculations, providing nano-object dimensions and orientation in excellent agreement with those determined from the TEM image of the nanorod (figure 3d).

The details of local surroundings play a much greater role for non-encapsulated nanoobjects. Recently, $\Omega_{R}$ values measured for bare gold nanorods and nanobipyramids deposited on TEM grids were compared to those computed using FEM modeling $[62,69]$. In this analysis, TEM-determined sizes were used as an input and TEM substrate was included. 
The use of a refractive index of $n_{\text {sur }}=1$ for the surrounding medium above the substrate (figure 3a) led to strong discrepancies between measured and simulated $\Omega_{R}$ values [69]. Conversely, excellent agreement was restored by adjusting $n_{\text {sur }}$ value (e.g., $n_{\text {sur }} \approx 1.4$ for gold nanorod experiments) [62] or surrounding the nano-objects by a water droplet in the modeling [69]. This suggests the presence of residual surfactant molecules or solvent (and possibly other impurities) around deposited nanoparticles. This hypothesis is supported by the large variations of $n_{\text {sur }}$ between differently synthesized colloidal solutions. For instance, $n_{\text {sur }}$ values much closer to $1(\approx 1.1)$ were found for nanobipyramids originating from solutions having undergone intense purification procedures [62]. Additionally, $n_{\text {sur }}$ fluctuations have been observed even for spherical [61] and elongated [62] nano-objects from the same solution. These findings stress the impact and variability of local environment (and notably the presence of surfactant molecules or residual solvent) on the SPR properties of bare nano-objects synthesized by chemical methods. They demonstrate that encapsulation is a useful way to provide them with a very well controlled environment, yielding quantitatively predictable SPR optical responses. In a reverse way, as the validity of classical modeling approaches based on Maxwell's laws has been experimentally established, such experimental and theoretical comparisons can also be used to obtain information on the intrinsic characteristics of individual nanoparticles and of their environment.

\subsection{Investigating single nanoparticle SPR damping: quality factor and quantum}

\section{confinement effects}

A. Bulk-like plasmon damping. In the case of intermediate-sized nano-objects such as gold nanorods with $\approx 15-20 \mathrm{~nm}$ diameter, $\Gamma$ is dominated by the bulk-like term $\Gamma_{0}=$ $\Omega_{R}{ }^{3} \varepsilon_{2}^{(b u l k)}\left(\Omega_{R}\right) / \omega_{p}^{2}$ (equations 7 and 8 ), enabling its wavelength dependence to be directly investigated (figure 4). Finite-element simulations performed in this regime (i.e., with $\gamma_{s}=0$ 
and on small nano-objects, so that $\Gamma_{r} \rightarrow 0$ ) confirm that this relation, computed above in the case of nanoellipsoids, holds for various nano-object shapes (nanoellipsoids, nanorods and nanobipyramids, as shown in figure 4a) [62]. For gold, combining this formula with tabulated bulk dielectric functions yields a quasi-constant $\Gamma_{0}$ value for photon energy below the interband threshold (occurring at $650 \mathrm{~nm}$ wavelength at the $X$ point of the Brillouin zone $[7,78]$ ), in agreement with the $\varepsilon_{2}^{(b u l k)} \propto \Omega_{R}^{-3}$ (equation 4) dependence predicted by Drude model. On the contrary, $\Gamma_{0}$ strongly increases below $650 \mathrm{~nm}$ wavelength, due to metal absorption enhancement by interband transitions (see figure 4a). Concomitantly, SPR shape becomes asymmetric in this spectral domain (as shown in the insets of figure $4 a$, and experimentally observed for gold nanospheres $[30,61,63]$ or weakly elongated nanorods [65]), the condition leading to a Lorentzian lineshape of the extinction spectrum (namely reduced variations of $\varepsilon_{2}$ in the SPR spectral range, see discussion before equation 6) not being fulfilled. This induces some ambiguity in the definition of SPR width (for instance, values determined from SPR spectra using either a Lorentzian interpolation or a direct measurement of half-width at half-maximum [30] do not coincide).

Figure $4 \mathrm{~b}$ presents the SPR widths measured on glass-deposited Au nanorods, embedded in an index matching layer (polymer or fluid) providing them with an optically uniform environment $[14,15,50,65,79]$. Intermediate-sized nanorods with a $13-20 \mathrm{~nm}$ diameter and 40-60 $\mathrm{nm}$ length were selected to draw this figure, on the basis of either average dimensions of their originating sample or of the optical determination of their size (electron microscopy being incompatible with embedded nano-objects). $\Gamma$ measurements performed in four different groups appear in excellent agreement with each other, and are also similar for bare and silica-encapsulated [79] nanorods. These results demonstrate the 
superior quality factor $\mathrm{Q}=\Omega_{R} / \Gamma$ offered by the SPR of elongated gold nanorods, as compared to lower aspect ratio and spherical gold nanoparticles. Moreover, they are also in good agreement with the $\Gamma_{0}$ expression above, with a relative dispersion of the order of the uncertainty on gold dielectric function (the predictions associated to three different $\varepsilon_{2}^{(b u l k)}$ tables being plotted in figure $4 \mathrm{~b})$. This confirms that the values and spectral variations of SPR width mostly follow those of $\Gamma_{0}$ for this nanorod size range.

Doping a metal nano-object with impurities composed of a highly dissipative material (i.e., with large $\varepsilon_{2}$ values) leads to a broadening of its SPR. This was evidenced by scattering measurements on single Au nanoshells doped with cobalt, for which a substantial increase of SPR width proportional to the cobalt fraction was observed [52]. An absorbing environment has also been shown to increase the damping rate of surface plasmons, as demonstrated for instance by the large increase of SPR width measured for metal nanostructures lithographed on top of a titanium adhesion layer [80] or deposited on a carbon substrate [76]. The magnitude of the induced SPR broadening depends on the penetration of the intense electromagnetic fields generated around a nano-object in the absorbing parts of its environment, and is thus strongly affected by both its shape and three-dimensional orientation, as recently demonstrated by correlating the measured and simulated extinction spectra of gold nanobipyramids on carbon substrates with the three-dimensional characterization of their orientation using electron tomography [76].

The effect of temperature on $\Gamma_{0}$ was also investigated through experiments of intermediate-sized (30 nm diameter, $90 \mathrm{~nm}$ length) gold bipyramids. An up to $30 \%$ narrowing of SPR with decreasing temperature was observed, the temperature dependence 
of SPR width reflecting that computed for the bulk-like optical scattering rate $\gamma_{0}$, mostly resulting from its electron-phonon component [81].

B. Radiative plasmon damping. Radiative damping $\left(\Gamma_{r}\right.$ term in equation 8$)$ induces significant SPR broadening as the size of the nano-objects increases above typically $30-40 \mathrm{~nm}$ [82], and increases linearly with volume $\left(\Gamma_{r}=\kappa V\right)$ in the quasistatic approximation, as analytically shown for nanoellipsoids (equation 9 in [83]; note that the proportionality between $\Gamma_{r}$ and $V$ is obtained only when the nanoparticle is described using the bulk dielectric function of its constituting metal, and is therefore not valid in the very small size regime [83], where this dielectric function is modified by the electron confinement effects described in the next paragraph). This trend was experimentally observed as a linear increase of $\Gamma$ with $V$ in a SEM-correlated study on large $(\approx 100 \mathrm{~nm}$ side length) silver nanoprisms [72], yielding $\kappa \approx 1.6$ $10^{-6} \mathrm{eV} \cdot \mathrm{nm}^{-3}$. Smaller $\kappa$ values $\kappa \approx 5-710^{-7} \mathrm{eV} \cdot \mathrm{nm}^{-3}$ were deduced from experiments on gold nanorods with lengths up to $100 \mathrm{~nm}[14,84]$. These results are however not incompatible, $\kappa$ depending on resonance wavelength and environment [83]. For this reason, the contribution to radiative broadening of the SPR of a single nano-object of arbitrary but known morphology can be accessed by numerically computing its SPR spectrum using bulk metal dielectric function (with thus $\gamma_{s}=0$ ). $\Gamma_{r}$ is thus obtained by subtracting the bulk contribution $\Gamma_{0}$ from the computed $\Gamma$ (equation 8) [79]. The results of such $\Gamma_{r}$ estimation are presented below for encapsulated gold nanorods (see figure $6 \mathrm{~b}$ ).

$\Gamma_{r}$ becomes the dominant contribution to SPR width for very large nano-objects (with dimensions exceeding $100 \mathrm{~nm}$ ). In this regime, $\sigma_{\text {ext }}$ spectra are not Lorentzian as they lump the contribution of various multipolar, spectrally overlapping plasmon modes [70]. Additionally, the $\Gamma_{r} \propto V$ relation established for dipolar SPR in the quasistatic regime is not 
valid anymore when retardation effects play an important role. Currently, many singleparticle studies on large nano-objects address radiative damping engineering, using the ability of plasmonic coupling to create subradiant and superradiant plasmon modes (i.e., modes with a different coupling efficiency with light, and thus different $\Gamma_{r}$ values) and induce Fano resonances (see section 3.3) in their scattered light. Different nanosystems have already been investigated, including nanodimers formed by two differently sized or shaped nano-objects [85], dolmen and non-concentric ring structures $[86,87]$ or deposited nanoparticles (e.g., cubes) in strong electromagnetic interaction with their substrate [88]. A detailed review of these effects is beyond the scope of this review focused on small isolated nano-objects.

C. Surface-mediated plasmon damping. Quantum confinement effects play a major role for small nano-objects (e.g., spheres with $<25 \mathrm{~nm}$ diameter). In the case of nanospheres, the surface broadening contribution $\left(\gamma_{s}\right)$ to the SPR width is predicted by both classical and simple quantum theories (e.g., modeling nanoparticle as an infinite potential well) [73] to be inversely proportional to diameter $D$ :

$$
\gamma_{s}=2 g_{s} \frac{v_{F}}{D}
$$

where $v_{F}$ is the metal Fermi velocity and $g_{s}$ a parameter of the order of unity. In the much more complex case of non-spherical nanoparticles, its description is still an open issue. In the simplest way, one can assume a similar expression for $\gamma_{s}$ by introducing an effective confinement length $L_{\text {eff: }}$

$$
\gamma_{s}=A \frac{v_{F}}{L_{e f f}}
$$


A major current challenge in the field of plasmonics is to quantify the magnitude of this effect ( $g_{s}$ and $A$ values) and to understand the dependence of $L_{\text {eff }}$ on nano-object dimensions, especially for elongated nano-objects such as nanorods characterized by two different length scales, their length $L$ and diameter $D$. Indeed, available models provide opposite results regarding the longitudinal SPR width of these nano-objects: classical "billiard" models [89] predict $L_{\text {eff }} \approx D$ (associated to an electronic mean free path limited by the short nanorod axis), while $L_{\text {eff }} \sim L$ (associated to a longitudinal electronic motion induced by light polarized parallel to the nanorod long axis) is expected according to simple quantum-based models [90].

Note that equations 9 and 10 above are expected to hold only for smooth, welldefined nanoparticle/environment interfaces. In practice, the presence of molecules at the surface of chemically synthesized nanoparticles may considerably modify the electronic density of states, inducing supplementary nonradiative decay channels for surface plasmons (i.e., additional electronic transitions available for Landau damping, as compared for instance to a metal nanoparticle in vacuum), a process referred to as "interface chemical damping" [91]. With this respect, recent ensemble measurements have demonstrated a large variation of SPR width between samples of small $(<12 \mathrm{~nm})$ silver nanoparticles synthesized using different stabilizing agents [92,93].

In the last years, much insight has been gained from single-particle investigations regarding the quantitative measurement of surface-broadening effects $\left(g_{\mathrm{s}}\right.$ and $A$ coefficients in equations 9 and 10) and, for non-spherical nano-objects, their morphology dependence ( $L_{\text {eff }}$ in equation 10$)$. In the following, we will first discuss results obtained on silica-coated metal nanospheres, and then those on elongated nano-objects. 
Size-dependent studies of SPR width have been performed for silver quasi-spherical nanoparticles, demonstrating resonance broadening for diameters $<25 \mathrm{~nm}$ [66]. The main results are shown in figure 5 . Silica encapsulation of the nanoparticles was used to protect them from oxidation and prevent any spurious interface damping mechanism. Additionally, the coated nanoparticles were embedded in a polymer layer to provide them with an optically quasi-uniform environment. Experimental extinction spectra of individual nanoparticles were quasi-Lorentzian in the energy domain (as expected from the spectral separation of nanosphere SPR and interband transitions in the UV domain for silver) with a slight polarization dependence because of small nanoparticle ellipticity. They were well reproduced using Mie theory, modeling the nanoparticle as a prolate ellipsoid and using a uniform 1.45 refractive index for the nanoparticle environment (figure $5 b$ ). In this analysis, nanoparticle dimensions (long and short axes $a$ and $b$ or, alternatively, aspect ratio $\eta=b / a$ and equivalent diameter $D_{e q}=2 \eta^{2 / 3} a$ ) and $\gamma_{s}$ (equations 8-10) were used as free parameters (figure 5b). The nanoparticle dimensions deduced from such a direct fit (Figure 5b) weakly depend (typically $<10 \%$ ) on the set of dielectric constants used (e.g., Johnson and Christy or Palik tables) [66], as a result of moderate uncertainties on silver $\varepsilon_{1}$. The same volumes are extracted by directly measuring the experimental spectral area of the quasi-Lorentzian silver SPR, using its $\Xi \propto V$ dependence [66]. Conversely, the $\gamma_{s(f i t)}$ values extracted from a direct reproduction of each SPR spectrum strongly depend on the set of dielectric constants used in the fitting process: for instance, an optimal reproduction of the SPR width in figure $5 \mathrm{~b}$ is obtained for $g_{s}$ values of 1.8 and 0.4 using Johnson and Christy or Palik data, respectively (figure 5c). This large discrepancy results from the limited experimental precision on silver bulk $\varepsilon_{2}$ (and thus $\Gamma_{0}$, see equations $7-8$ ), due to its small value in the visible range. Writing the bulk damping rate used in the fit as $\Gamma_{0(f i t)}=\Gamma_{0^{+}}+\Delta \Gamma_{O(t a b)}$, i.e., the sum of its physical value 
and an error due to uncertainties in tabulated dielectric functions, and neglecting radiative damping in this low-size regime (so that $\Gamma=\Gamma_{0}+\gamma_{s}=\Gamma_{0(f i t)}+\gamma_{s(f i t)}$ ), it appears that $\gamma_{s(f i t)}=\gamma_{s}-\Delta \Gamma_{O(t a b)}$. Therefore, optimal $\gamma_{s(f i t)}$ fitting values include corrections to errors in $\Gamma_{0}$ unrelated to surface-related damping processes, which prevents a reliable extraction of $\gamma_{s}$ from individual extinction spectra of silver nanoparticles by direct spectrum fitting.

This information can however be obtained by correlating the sizes and SPR linewidths measured for different nanoparticles, i.e., using size-induced interparticle variations of $\Gamma$ rather than its absolute value. Experimentally, $\Gamma$ was found to vary linearly with $1 / D_{e q}$ in the low size regime, in agreement with equations 8 and 9. The measured slope thus permits a direct full experimental determination of the surface factor, yielding $g_{s}=0.7 \pm 0.1$. Extrapolation of the linear dependence observed in the low size regime $\left(1 / D_{e q} \rightarrow 0\right)$ yields $\Gamma_{0}=125 \pm 10 \mathrm{meV}$. This value stands between those associated to Johnson and Christy (60 $\mathrm{meV}$ ) and Palik (230 meV) measurements (see figure 5c), confirming the large uncertainties associated to these values. In more detail, from a theoretical point of view the above analysis overestimates the value of pure surface scattering, as $\gamma_{s}$ lumps all possible size effects on electron scattering mechanisms (i.e., modifications of phonon and electron scattering rates included in $\Gamma_{0}$ due to size reduction). A corrected value of the pure surface scattering coefficient $g_{\mathrm{s}}$ can be obtained by estimating the size variations of electron-phonon scattering rate, yielding $g_{\mathrm{s}} \approx 0.45 \pm 0.1[66]$.

These experimental results were subsequently confronted to numerical calculations based on a time-dependent local density approximation [43], extending the pioneering work of Kawabata and Kubo [73]. In this work, a jellium approach ignoring the granularity of the ionic lattice was used in combination with a self-consistent calculation of electronic 
confinement potential using Kohn-Sham formalism (in contrast with the approximation of an infinite confinement potential made in earlier calculations). Nanosphere absorption was deduced using standard theory of linear optical response. Figure 5a illustrates the most important results obtained for silver nanospheres using this complete quantum approach. The $\gamma_{s} \propto 1 / D$ scaling law predicted by classical approaches $[89,94,95]$ and by Kawabata and Kubo's simplified quantum calculations [73] is confirmed for $>5 \mathrm{~nm}$ large nanospheres. Extending initial calculations where environment was not taken into account [73], this model reveals a strong dependence of confinement effects on the dielectric medium surrounding the nanospheres, $g_{s}$ increasing with the environment refractive index. In particular, the value computed for nanospheres in silica $g_{\mathrm{s}}=0.32$ is in reasonable agreement with the $0.45 \pm 0.1$ one extracted from experiments after deduction of other size-dependent effects.

A smaller $g_{\mathrm{s}}$ value was suggested in a previous study performed on single gold nanospheres [30]. However, the asymmetric shape of gold nanosphere SPR (strongly influenced by interband transitions), as well as the less detailed information about nanoparticle size (optical determination of individual nanoparticle sizes being not possible in the context of photothermal spectroscopy) complicated the analysis of this earlier study. When taking into account the associated uncertainties, the SPR width size-dependence of the small gold nanoparticles remains however compatible with the lower range of $g_{\mathrm{s}}$ values estimated in our studies on silver spheres [66] and gold nanorods [79] (see below).

Understanding quantum confinement effects in the case of non-spherical nanoparticles is particularly important, as demonstrated by the large interest for optical investigations of gold nanorods at the single-particle level $[14,15,50,65,79]$. Figure $6 a$ summarizes SPR width measurements performed in different groups on gold nanorods with 
various sizes embedded in an index-matching medium. Two types of data points are presented in this figure, corresponding to SMS measurements (enabling optical determination of dimensions for each nanorod) [79] and to sample-averaged data on nanorod solutions obtained with non-quantitative techniques (errors bars representing standard deviations of nano-object dimensions of the samples) $[14,15,50]$. The agreement between the data of different groups previously mentioned for the population of mediumsized rods discussed above (figure 4) remains excellent for the extended dimension range of figure 6a. This plot demonstrates a dependence of $\Gamma$ on both $L$ and $D$, with a drastic $\approx 60 \mathrm{meV}$ increase for the smallest nano-objects ( $L \approx 30 \mathrm{~nm}$ and $D \approx 7 \mathrm{~nm}$, see also figure $3 b$ ) as compared to the largest ones $(L \approx 70 \mathrm{~nm}$ and $D \approx 20 \mathrm{~nm})$. The estimated contributions of bulk and radiative damping to these variations are weak, $\Gamma_{0}$ and $\Gamma_{r}$ being almost constant for the vast majority of the investigated nanorods (with only an increase of $\Gamma_{0}$ for the smallest aspect ratios, as discussed previously (Figure 4), and of $\Gamma_{r}$ for the largest ones) (figure 6b). Variations are therefore mostly due to quantum confinement effects.

Their dependence on $L$ and $D$ strongly differ from those predicted by simple quantum model $\left(\gamma_{s} \propto 1 / L[90]\right)$ and classical billiard approaches $\left(\gamma_{s}\right.$ scaling with $1 / D$ for elongated nano-objects [89]) (figure 6c). SMS results show that it is empirically well reproduced by the $\gamma_{s} \propto 1 /\left(L^{1 / 2} D^{1 / 2}\right)$ relation, giving the same weight to $L$ and $D$ (figure $6 \mathrm{c}-\mathrm{d}$ ). This suggests a new geometrical parameter controlling quantum confinement effects, namely the nano-object surface $S(S=\pi L D$ for a hemispherically-capped nanorod), yielding an effective scattering length $L_{\text {eff }}=(S / \pi)^{1 / 2}$ (equation 10$)$ and $A=1.4$. These results obtained for gold nanorods also permit a quantitative interpretation of surface broadening in silver spheres (for which $L_{\text {eff }}=D$ ) with the same surface factor $\left(g_{s}=A / 2=0.7\right)$. The amplitudes of surface effects predicted by 
this empirical formula are also compatible with those measured for different nano-object shapes, e.g., oblate nanoprisms $[71,72]$. This further points out the importance of surface area as a relevant parameter for describing quantum confinement effects in nano-objects. However, importantly, its expected range of validity does not include all nano-object shapes. For instance, this simple approach is not expected to be valid for non-convex shapes such as nanoshells [96]. Indeed, comparing a nanosphere and a thin nanoshell of same external radius, surface is larger in the second case while quantum confinement should be more pronounced due to the small thickness. This stresses the difficulty of simple theoretical models to yield truly general results, as well as the necessity of building complete quantum models for each nano-object shape of interest.

Electron-surface scattering processes strongly depend on the interface between the nanoparticles and their environment (e.g., number and nature of bound molecules), resulting in a strong dependence of SPR width on nanoparticle synthesis procedures. The magnitude of this additional interface broadening has been found to be most intense for surfactant-stabilized nanoparticles deposited on a substrate $[62,97]$, while usually negligible for silica-encapsulated nano-objects [66,79]. Additionally, experiments suggest a marked dependence on nano-object shape, more pronounced effects having been observed for spheres than for rods. As mentioned above, this effect plays a negligible role on the SPR widths of polymer-embedded and silica-encapsulated gold nanorods [14,50,65,79,84]. Conversely, extra-broadening has been observed for surfactant-coated rods deposited in air on a substrate. In particular, Zijlstra et al. recently investigated the effect of thiol coating on the SPR linewidth of gold nanorods by comparing the effect of no, tip-specific and full functionalizations on the same nanorods [98]. They found a linear relation between SPR broadening (with a $\Gamma$ increase of $\approx 30 \mathrm{meV}$ for full functionalization) and thiol density on 
nanorod surface. These results are consistent with those obtained on deposited nanorods and bipyramids [62]. Indeed, analysis of these experiments leads to $\gamma_{s}$ values in the $50-100$ meV range, exceeding by up to 50 meV the predictions of equation 10 used with $A=1.4$ and $L_{\text {eff }}=(S / \pi)^{1 / 2}$. Much stronger chemical damping effects have been observed in surfactantstabilized, polymer-embedded gold nanospheres [97]. In particular, large ( $>100 \mathrm{meV})$ interparticle variations of $\gamma_{s}$ were observed, in stark contrast with experiments on polymerembedded $\mathrm{Ag} @ \mathrm{SiO}_{2}$ nanospheres (figure 5), $\mathrm{Au}$ and $\mathrm{Au} @ \mathrm{SiO}_{2}$ nanorods (figure 6) and deposited gold nanorods and bipyramids[62] ( $<50 \mathrm{meV}$ deviations from mean width). Understanding the magnitude, shape and environment dependences of these interface effects will require extensive quantum modeling of the coupling between nanoparticle and surfactant electronic states [92].

\section{Ultrafast plasmonics of individual nano-objects}

Additional understanding of important physical processes in metals (e.g., electronic and lattice dynamics) and of their modification at the nanoscale can be obtained by ultrafast time-resolved experiments $[46,99-101]$. Such dynamical processes can be induced by the impulsive excitation of a metal nano-object by a femtosecond pulse (pump). This leads to a time-dependent modification of its optical response (e.g. $\sigma_{\text {ext, }}$ see equation 5 for an elongated or spherical nano-object), which can be followed using a second pulse (probe, delayed by time $t_{D}$ with respect to the pump) interacting with the sample. In the case of an individual nano-object, neglecting at short time scale environment heating (leading to variation of $\varepsilon_{m}$ described in $\left.[102,103]\right)$ and transient shape deformations induced by acoustic vibrations (e.g. inducing change of $\left.L_{i}[10]\right)$, the modifications of its optical extinction cross 
section at probe wavelength $\lambda_{p r}=h c / \omega$ can be linked to the time-dependent variations of the metal dielectric functions by:

$$
\begin{aligned}
\Delta \sigma_{e x t}\left(\lambda_{p r}, t_{D}\right) & =\frac{\partial \sigma_{e x t}}{\partial \varepsilon_{1}}\left(\lambda_{p r}\right) \Delta \varepsilon_{1}\left(\lambda_{p r}, t_{D}\right)+\frac{\partial \sigma_{e x t}}{\partial \varepsilon_{2}}\left(\lambda_{p r}\right) \Delta \varepsilon_{2}\left(\lambda_{p r}, t_{D}\right) \\
& =a_{1}\left(\lambda_{p r}\right) \Delta \varepsilon_{1}\left(\lambda_{p r}, t_{D}\right)+a_{2}\left(\lambda_{p r}\right) \Delta \varepsilon_{2}\left(\lambda_{p r}, t_{D}\right)
\end{aligned}
$$

with $a_{1,2}$ the static spectral-dependent $\sigma_{e x t}$ derivatives and $\Delta \varepsilon_{1,2}$ the modifications of real and imaginary parts of $\varepsilon$ including both interband $\left(\Delta \varepsilon_{1,2}^{i b}\right)$ and intraband (Drude-like, $\Delta \varepsilon_{1,2}^{D}$ ) contributions $[7,46]$ (equation 4). Equation 11 thus allows to split the analysis of the ultrafast response of a metal nano-object in two parts. Plasmonic local field effects are described by the stationary terms $a_{1,2}$, which display large enhancements around the SPR, while the kinetical response induced by impulsive heating is described by the temporal variations of the metal dielectric functions.

In the following, we will focus on optical experiments and quantitative modeling of transient absorption spectra in single metal nano-objects (gold nanorods and silver-gold nanodimers), in order to investigate their ultrafast electronic response. The vibrational dynamics of individual nanoparticles can also be efficiently investigated by ultrafast plasmonics, but its discussion is out of the scope of this review (see for instance $[10,104]$ ).

\subsection{Femtosecond spectroscopy of individual nanoparticles: nanospheres and nanorods}

In the case of a silver nanosphere in a homogeneous environment (figure 7a), spectral separation between plasmonic resonance (around $420 \mathrm{~nm}$ ) and interband transitions (below $350 \mathrm{~nm}$ ) reflects into a sharp SPR peak, which turns into spectrally narrow and enhanced $a_{1,2}$ derivatives. The broader SPR of a gold nanosphere, partially overlapping interband transitions (figure $7 \mathrm{~b}$ ), reflects into spectral variations of $a_{1,2}$ on a more extended 
range around $530 \mathrm{~nm}$. As expected, modification of a nano-object shape from a spherical to an elongated geometry (nanorod) induces a spectral red-shift of its longitudinal SPR resonance and consequently on the resonances of its $a_{1,2}$ derivatives (figure $7 b, c$ ).

The dynamics of $\Delta \varepsilon_{1,2}$ in equation 11 is expected to be dominated by interband terms $\Delta \varepsilon_{1,2}^{i b}$ at short time scale (see section 3.2). These contributions come from the modification of the probability of transitions from occupied inner electronic states to the conduction band around the Fermi energy following electron heating. Electron occupation number $f\left(E, t_{D}\right)$ for the quasi-free electron gas is characterized at thermal equilibrium by a Fermi-Dirac distribution, its evolution with time being described by the Boltzmann equation $[7,46,99]$ :

$$
\frac{\partial f\left(E, t_{D}\right)}{\partial t}=\left.\frac{\partial f\left(E, t_{D}\right)}{\partial t}\right|_{e x c}+\left.\frac{\partial f\left(E, t_{D}\right)}{\partial t}\right|_{e-e}+\left.\frac{\partial f\left(E, t_{D}\right)}{\partial t}\right|_{e-p h}
$$

where the three terms specify $f\left(E, t_{D}\right)$ modifications induced by pump pulse excitation, electron gas internal thermalization by electron-electron scattering and electron energy loss to lattice (phonons), respectively. After solution of the Boltzmann equation, $\Delta \varepsilon_{2}^{i b}\left(\lambda_{p r}, t_{D}\right)$ can be computed from $\Delta f\left(E, t_{D}\right)$ using a band structure model [105], by integrating, for all the bound electron initial energy states, the probability of transition from occupied to quasi-free unoccupied states with an energy difference equal to the probe photon energy. The corresponding real part $\Delta \varepsilon_{1}^{i b}$ can be deduced by Kramers-Kronig integral transformation of transient $\Delta \varepsilon_{2}^{i b}$ [46]. While this approach was first introduced to compute the kinetics of bulk metals, it can be extended to noble metal nanoparticles with sizes $>2-3 \mathrm{~nm}$, provided sizedependent modifications of the electron-electron [106] and electron-phonon coupling [107] are taken into account. 
Other contributions to $\Delta \varepsilon_{1,2}$, both on short and longer timescales, come from modifications of the Drude intraband terms, $\Delta \varepsilon_{1,2}^{D}$, which are affected by both electronic and lattice heating. We focus our discussion here on the dominant effects (see for instance [7] for a complete discussion). Hot electron distribution alters $\gamma_{e-e}$ in the $\gamma_{0}$ optical scattering rate of conduction electrons (section 2.2), thus modifying $\Delta \varepsilon_{2}^{D}=\Delta \gamma_{0} \omega_{p}^{2} / \omega^{3}$ (equation 4). It also modifies $\gamma_{s}$, the electron-surface scattering term (through modifications of $g_{s}$ or $A$ factors, equations 9 and 10), but this contribution is negligible except for very small (few $\mathrm{nm}$ ) particles [7]. Heating of the lattice at a temperature $T_{L}=T_{0}+\Delta T_{L}$ by energy transfer from the excited electrons leads to both an increase of the $\gamma_{e-p h}$ term (contributing to $\Delta \varepsilon_{2}^{D}$ ) and to a reduction of plasma frequency $\omega_{p}$ (affecting $\Delta \varepsilon_{1}^{D}$ ) induced by particle thermal dilation.

Equation 11 quantitatively links the ultrafast optical response of a metal nanoparticle to these out-of-equilibrium phenomena. Femtosecond spectroscopy of a single nano-object combined to this modeling is an appropriate tool to investigate such ultrafast electronic processes, as shown below in the case of single nanorods.

\subsection{Ultrafast optical nonlinearities in confined metals: physical origin and quantitative}

\section{contributions}

Femtosecond pump-probe spectroscopy on a single nanorod (length $L=43 \mathrm{~nm}$ and diameter $D=12 \mathrm{~nm}$ ) deposited on a transparent substrate was performed by illuminating it with two focused pump $\left(\lambda_{p p}\right)$ and probe $\left(\lambda_{p r}\right)$ femtosecond pulse trains [44]. Due to the required very high sensitivity, a high repetition rate system was used based on a Ti:Sapphire laser generating $\approx 150 \mathrm{fs}$ pulses tunable in the $760-860 \mathrm{~nm}$ range. Pump pulses in the 380 $430 \mathrm{~nm}$ wavelength range were generated by frequency doubling a part of these near- 
infrared pulses in a nonlinear BBO crystal. For the experiment on heterodimers described in section 3.3, 530-550 $\mathrm{nm}$ probe pulses were also generated by a visible Optical Parametric Oscillator pumped by part of the Ti:Sapphire laser.

A quantitative linear optical characterization was first performed by SMS, providing the nanorod extinction spectrum $\sigma_{\text {ext }}$. Sample mechanical modulation was then stopped and pump and probe beams were both focused on the nanorod using the same SMS microscope objective. The transient sample transmission change is directly related to the induced modifications of the nano-object extinction cross-section: $\Delta T / T=-\Delta \sigma_{e x t} / S_{p r}$, where $T$ is the probe beam transmission and $S_{p r}=\pi \Phi_{p r}^{2} /(4 \ln 2)$ its focused spot size (full-width-athalf-maximum of a Gaussian focal spot).

Experimental $\Delta \sigma_{e x t}\left(\lambda_{p r}\right)$ are shown in figure 8g-i (blue dots) at three different pumpprobe delays $t_{D}=0 \mathrm{fs}, 200 \mathrm{fs}$ and $4 \mathrm{ps}$. These values are compared to the $\Delta \sigma_{\text {ext }}$ computed following the model described above. This includes solution of Boltzmann equation and band structure model for the determination of interband contributions $\Delta \varepsilon_{1,2}^{i b}$, and estimation of intraband terms $\Delta \varepsilon_{1,2}^{D}$ from electron and lattice heating (figure 8a-c). $\Delta \sigma_{e x t}$ variations coming from interband (intraband) term are subsequently computed (figure $8 \mathrm{~d}$-f) by combining $\Delta \varepsilon_{1,2}^{i b}\left(\Delta \varepsilon_{1,2}^{D}\right)$ and $a_{1,2}$ of the investigated nanorod (equation 11 , figure $\left.7 \mathrm{c}\right) . \Delta \gamma_{\text {e-e }}$ contribution to $\Delta \varepsilon_{2}^{D}$ can be estimated using the formula $\Delta \gamma_{e-e} / \gamma_{e-e}=\left(2 \pi k_{B} / \hbar \omega\right)^{2}\left(T_{e}^{2}-T_{0}^{2}\right)$, where $T_{e}$ is the equivalent electron temperature of a thermalized electron gas with same excess energy, and $T_{0}$ the initial equilibrium temperature $[108,109]$. This contribution depends quadratically on $T_{e}$ and is therefore expected to be dominant at short timescale and in the case of strong electron excitation. On the other hand, the increase of the $\gamma_{e-p h}$ term concomitant with 
lattice heating, which is a consequence of the increase of the phonon occupation number with lattice temperature, is proportional to lattice temperature increase $\Delta T_{L}$, with $\Delta \gamma_{e-p h} / \gamma_{e-p h} \approx \Delta T_{L} / T_{0}$. Rough estimation of optical scattering rates $\gamma_{e-e}$ and $\gamma_{e-p h}$ in the infrared range can be obtained from their total value $\hbar \gamma_{0}=\hbar \gamma_{e-e}+\hbar \gamma_{e-p h}$, assuming that $\gamma_{e-e} / \gamma_{0}$ and $\gamma_{e-p h} / \gamma_{0}$ are approximately $10 \%$ and $90 \%$ respectively [110].

In single particle experiments, quantitative knowledge of the nano-object linear absorption permits a precise determination of the optical excitation, yielding an equivalent initial electron temperature increase $\Delta T_{\text {exc }} \approx 125 \mathrm{~K}$ for the used experimental pump energy fluence. As expected, $\Delta \varepsilon_{1,2}^{i b}$ terms are dominant at the very short timescales ( $t_{D}=0 \mathrm{fs}$ and $200 \mathrm{fs}$ ) (figure 8a, b), the computed $\Delta \sigma_{\text {ext }}$ being approximately equal to its interband contribution $\Delta \sigma_{\text {ext }}^{i b}$ (figure $8 \mathrm{~d}$, e). As the interband ultrafast components directly reflect the out-of-equilibrium modifications $\Delta f$ of the electron occupation number, their values are strongly reduced at longer delays (4 ps, figure $8 \mathrm{c}$ ), energy being transferred to the lattice with a time constant of $1.1 \mathrm{ps}$ in gold [107]. For short delays, the intraband term $\Delta \sigma_{e x t}^{D}$ is only affected by the electronic $\Delta \gamma_{\text {e-e }}$ contribution to $\Delta \varepsilon_{2}^{D}$, which is however small as compared to interband contributions (figure $8 \mathrm{a}, \mathrm{d})$. For $t_{D}=4 \mathrm{ps}$, heating of the lattice $\left(\Delta T_{L} \approx\right.$ $1 \mathrm{~K}$ ) concomitant with electron cooling modifies the dominant contributions to the transient optical signal. Intraband terms increase due to increase of the optical electron-lattice scattering rate $\Delta \gamma_{e-p h}$ (inducing $\Delta \varepsilon_{2}^{D}$ ), and via particle thermal dilation (inducing $\Delta \varepsilon_{1}^{D}=3 \alpha_{L} \Delta T_{L} \omega_{p}^{2} / \omega^{2}$, with $\alpha_{L} \approx 1.42 \times 10^{-5} \mathrm{~K}^{-1}$ being the Au linear dilation coefficient) (figure 8c). These two terms affect $\Delta \sigma_{\text {ext }}$ equivalently (figure $8 \mathrm{f}, \mathrm{i}$ ) and are the dominant ones at picosecond time scale (as also shown in experiments on single lithographed gold nanorods 
[111]). It should be noted that $\Delta \varepsilon_{1,2}$ contributions can also be directly measured using interferometric techniques, as demonstrated on single spherical gold nanoparticles by fourwave mixing microscopy experiments with heterodyne detection $[112,113]$.

As $\Delta \varepsilon_{1,2}^{i b, D}$ modifications are weakly dispersed in the infrared (figure 8a-c), ultrafast $\Delta \sigma_{\text {ext }}$ variations spectrally reflect a combination of $a_{1}$ and $a_{2}$, their relative weight being given by $\Delta \varepsilon_{1,2}$ and therefore varying with time. In particular, $a_{1}$ profile dominates the $\Delta \sigma_{e x t}$ response at short delays ( $t_{D}=200 \mathrm{fs}$ ), as the only significant contribution comes from $\Delta \varepsilon_{1}^{i b}$ (figure $8 \mathrm{~b}$ ). For $t_{D}=4$ ps (figure $8 \mathrm{c}$ ), $\Delta \varepsilon_{1}^{D}$ and $\Delta \varepsilon_{2}^{D}$ have equivalent amplitudes which manifest into a more complex $\Delta \sigma_{\text {ext }}$ shape. An excellent reproduction of single nanorod ultrafast nonlinear experiments is obtained using this modeling for all timescales (figure 8). It should be mentioned that significant approximations are made in the calculations of intraband contributions $\Delta \varepsilon_{2}^{D}$, as $\gamma_{e-e}$ and $\gamma_{e-p h}$ rates are not accurately known. Results in figure 8 are computed using $\hbar \gamma_{0}=20 \mathrm{meV}$ (value deduced from fitting the gold dielectric function of Johnson and Christy [7,114]). A different value $\hbar \gamma_{0}=(47 \pm 10) \mathrm{meV}$ could be used ( $\hbar \gamma_{e-e} \approx 5 \mathrm{meV}$ and $\hbar \gamma_{e-p h} \approx 40 \mathrm{meV}$ ), as estimated from more recent dielectric function measurements in gold [115], also consistent with SPR width measurements on individual nanorods (figure 6) and with temperature dependent experiments on bulk gold (providing $\left.\partial \hbar \gamma_{e-p h} / \partial T_{L} \approx 0.12 \mathrm{meV} / \mathrm{K}[109]\right)$. This modifies the corresponding extinction cross section variations at long time scale, yet remaining in good agreement with experimental data (figure 8g-i dashed lines).

Quantitative comparison between these optical time-resolved experiments on single nano-objects and theory proves that the origin of ultrafast nonlinearities in confined metals 
is the third order Kerr-like nonlinearity of the bulk metal (pump induced modification of its dielectric function), enhanced at frequencies close to their SPR due to plasmonic local field effect. The ultrafast response is dominated by interband transition modifications due to outof-equilibrium hot electrons. The wavelength dependence of these transient signals at femtosecond timescale reflects the frequency dispersion of the plasmonic coefficient $a_{1}=\partial \sigma_{e x t} / \partial \varepsilon_{1} \approx \omega^{3} /\left(2 \omega_{p}^{2}\right) \partial \sigma_{e x t} / \partial \omega$ [44] which is amplified around the SPR, with a maximum value proportional to $\approx \sigma_{e x t}^{\max } \Omega_{R} / \Gamma\left(\Omega_{R}\right.$ and $\Gamma$ being the spectral position and width of the linear extinction cross-section discussed in Section 2). This emphasizes the fact that nano-objects displaying large nonlinear changes of their extinction cross section are among the ones with the highest linear SPR quality factor, as elongated gold nanorods or bypiramids [62]. On a picosecond time scale, the ultrafast optical nonlinearity is a consequence of lattice heating, with the most significant contribution coming from modifications of the electron-phonon $\gamma_{e-p h}$ optical scattering rate.

\subsection{Ultrafast plasmonics of coupled nanoparticles: Fano effect in heterodimers}

Experiments performed using nonlinear time-resolved spectroscopy also provide a way to distinguish between the optical responses of a multi component nano-object like a bimetallic nanodimer, with a sub-wavelength spatial selectivity. By using two-color pumpprobe technique, light absorption of one of the dimer components can be selectively monitored, unveiling information otherwise hidden in the total linear optical response of the system. In this context, the first direct experimental evidence of the existence of Fano interference in the absorption of a nano-object has been recently demonstrated [45].

Fano resonance is present in the response of many systems in physics $[116,117]$. It has recently received a large attention in nano-photonics, and particularly plasmonics, as 
Fano interference between two plasmonic modes can induce narrow spectral features that can be exploited for designing ultrasensitive detectors $[87,88]$. Up to now, optical Fano resonances were investigated in large nanosystems and thus showed-up in their scattering spectra, corresponding to interferences between two spectrally overlapping modes of the full system. Fano resonance in the absorption of much smaller plasmonic objects, namely bimetallic heterodimers, was predicted few years ago but not demonstrated experimentally, due to difficulties in both a controlled material synthesis and in finding an appropriate experiment to unveil the phenomena [118]. In this context, dimers formed by a gold and a silver nanoparticle are model systems where Fano interference takes place between optical excitations of different nature: plasmonic and non-plasmonic ones. Furthermore, conversely to previously investigated atomic-, molecular-, or nanosystems, the involved modes mostly correspond to excitation of one or the other dimer components, introducing large versatility for tailoring the Fano resonance effect.

Individual gold-silver heterodimers were investigated to avoid orientation and morphology dispersions effects and to quantitatively compare the experimental and theoretical results (figure 9). The $\mathrm{Ag} @ \mathrm{SiO}_{2}-\mathrm{Au}$ dimers were synthesized by electrostatical assembly of gold nanospheres and silica-coated silver ones [3], and subsequently spincoated on silica membranes. The polarization-dependent extinction spectra of individual dimers were measured using spatial modulation spectroscopy, and their morphology precisely characterized using high magnification TEM after the optical experiments.

Numerical computations of the linear optical response were performed by finiteelement simulations using the TEM measured morphology of each individual dimer, taking into account their inhomogeneous environment (presence of a substrate), and when required, deviation from sphericity of the forming gold and/or silver nanoparticles (figure 9). 
Absorption cross-sections of the whole dimer (figure 9a) and of its gold and silver components were computed from the spatial integral of resistive heating over their respective volumes. As a direct consequence of gold-silver electromagnetic interaction, light absorption in one of the component (i.e., gold) is expected to be strongly altered by the vicinity of the silver nanoparticle, with a characteristic dispersed polarization-dependent spectral profile (i.e. Fano interference) around the silver plasmonic mode wavelength (figure 9b). This strong polarization dependence in the blue part of the spectrum is absent for an isolated gold nanosphere, even if it is slightly elliptical (absorption being dominated by interband transitions), and also for the whole dimer (for which absorption is dominated by the strong plasmonic response of its silver component, figure 9a).

Two-color pump-probe experiments were performed to selectively address and quantitatively probe the light energy absorbed by only one of the two constituting materials, the gold component. The whole dimer is excited by absorption of a pump pulse of wavelength $\lambda_{p p}$ in the $400-420 \mathrm{~nm}$ range that heats-up differently each component depending on its absorption cross-section within the dimer, $\sigma_{a b s}^{m}\left(\lambda_{p p}\right)$. In the low perturbation regime, for sufficiently low pump fluence $F_{p p}$, the increase of the electron temperature in the $m$ metal (either $\mathrm{Au}$ or $\mathrm{Ag}$ ) component is directly proportional to the excess energy injected into the system by absorption of the pump pulse:

$$
\Delta T_{e x c}^{m}\left(\lambda_{p p}\right) \approx \sigma_{a b s}^{m}\left(\lambda_{p p}\right) F_{p p} /\left(V_{m} c_{T}^{m}\right)
$$

where $V_{m}$ and $c_{T}^{m}$ are the metal volume and heat capacity. 
Heating of the gold component by pump absorption can be subsequently selectively monitored by a second time-delayed probe pulse at wavelength $\lambda_{p r}$. Its transient transmission change can be modeled by generalizing equation 11 to a bimetallic system:

$\Delta \sigma_{e x t}\left(\lambda_{p p}, \lambda_{p r}, t_{D}\right)=\sum_{m=A u, A g}\left[\frac{\partial \sigma_{e x t}}{\partial \varepsilon_{1}^{m}}\left(\lambda_{p r}\right) \Delta \varepsilon_{1}^{m}\left(\lambda_{p p}, \lambda_{p r}, t_{D}\right)+\frac{\partial \sigma_{e x t}}{\partial \varepsilon_{2}^{m}}\left(\lambda_{p r}\right) \Delta \varepsilon_{2}^{m}\left(\lambda_{p p}, \lambda_{p r}, t_{D}\right)\right]$

Similarly to the case of single nanoparticles (see figure $7 a, b$ ), the derivatives were obtained by numerical derivation of the computed dimer extinction cross-section, using the TEM measured dimer morphology. The transient variations of the dielectric functions of gold/silver components were quantitatively calculated by first determining the temporal evolution of electronic distribution through the resolution of Boltzmann equation and then deducing the resultant wavelength-dependent evolution of metal dielectric functions using Rosei band structure models [45].

By properly adjusting the probe wavelength close to the gold-like SPR, $\lambda_{p r}=530 \mathrm{~nm}$, contributions from the nonlinear response of gold dominate in equation 14, due to both plasmonic enhancement of the gold derivative coefficients and large interband gold dielectric function changes at this probe wavelength. At fixed time delay $t_{D}$ maximizing the transient response, the nonlinear signal therefore directly reflects the heating of the gold nanoparticle within the dimer, being a direct signature of its absorption cross-section:

$$
\left|\Delta \sigma_{e x t}\right|_{\max }=\frac{\left|\Delta \sigma_{e x t}^{r e f}\right|_{\max }}{\Delta T_{e x c}^{r e f}} \Delta T_{e x c}^{A u}\left(\lambda_{p p}\right) \propto \sigma_{a b s}^{A u}\left(\lambda_{p p}\right)
$$


where $\Delta \sigma_{e x t}^{r e f} / \Delta T_{e x c}^{r e f}$ is the extinction change at $\lambda_{p r}=530 \mathrm{~nm}$ induced by unitary increase of gold electronic temperature.

The experimental and computed extinction changes are shown in figure 9c,d for different pump wavelengths. A spectrally dispersed and polarization-dependent nonlinear response is measured, in excellent agreement with numerical modeling based on the gold parallel and orthogonal absorption cross-section shown in figure 9b. This experimentally demonstrates the presence of Fano interference in the spectral profile of gold absorption within the dimer. The amplitude of this Fano effect is tunable by changing the $\mathrm{Ag}-\mathrm{Au}$ component distance, suggesting the possibility to exploit these composite nanomaterials as optical analogs of a field-effect transistor (i.e. a variable electrical resistor: here, a variable optical absorber), where the response (the electron flux: here, photon absorption) to a signal (the source-drain voltage: here, light polarization direction) can be controlled by an external parameter (the voltage applied to the gate: here, the distance between the two nanoparticles composing the heterodimer, provided the dimers are synthetized with lengthtunable spacers such as molecular links undergoing thermal or optical conformational changes).

\section{Conclusion and perspectives}

In conclusion, single-particle optical techniques developed in the last years have provided important information on the optical and physical properties of nano-objects that would most probably have remained hidden in ensemble measurements. They appear to be complementary, as each of them present distinct advantages. While near-field microscopy has a superior resolution and allows direct imaging of electromagnetic field distributions, dark-field microscopy has the advantages of simplicity and wide-field imaging capabilities. 
Photothermal microscopy is extremely sensitive and compatible with scattering biological environments. Finally, SMS has the ability of measuring absolute extinction cross-sections, a crucial advantage for yielding fundamental physical insights. These techniques have shed light on the SPR properties (spectral position, area and width) of metal nano-objects. In most cases, the two first properties are well described using the classical laws of electromagnetism together with the dielectric constants of bulk materials. Conversely, in the low size regime, SPR width is significantly affected by electronic confinement effects, as well as by the nature of the interface between the nanoparticle and its environment. A complete picture of SPR is currently emerging for metal nanospheres, where quantum confinement effects have been characterized both experimentally and theoretically. However, much work remains to be done to understand the modalities of quantum confinement in non-spherical nano-objects.

Ultrafast experiments on a single metal nano-object have the advantage to permit a quantitative comparison with theoretical modeling. Femtosecond investigations on individual noble metal nanospheres and nanorods have elucidated the physical mechanisms at the origin of their optical nonlinearities upon electron excitation by absorption of a light pulse. These have been attributed to induced modifications of the metal dielectric function (with interband and intraband contributions as in bulk materials), enhanced by local field plasmonic effects when probing close to the SPR. The lattice dynamics, i.e. the acoustic vibrations of a metal nano-object, can also be efficiently investigated at single particle level, providing important information on the elastic properties of the nanoparticles and their local environment. Moreover, two-color pump-probe nonlinear spectroscopy can be usefully employed to selectively excite and monitor a part of a multicomponent nano-object by spectral selection. This versatility has demonstrated, in the case of a gold - silver 
heterodimer, the presence of a strong Fano resonance in the absorption on one of the two components under the influence of the other one. The polarization-dependent Fano effect is masked in the linear optical spectrum of the whole dimer, but can be evidenced using ultrafast plasmonics. This also opens the way to future single object optical investigations of charge and energy exchanges at nanoscale with a spatial selectivity much smaller than the optical wavelengths.

\section{Acknowledgments}

The authors would like to thank FemtoNanoOptics former students who contributed to single-particle and ultrafast investigations, in particular Hatim Baida, Vincent Juvé, Denis Mongin, Anna Lombardi, Jean-Christophe Blancon, Tatjana Stoll and Etienne Pertreux. Financial support of these researches by ANR programs TRI-CO under contract ANR-11NANO-025, UNIT-3D under contract ANR-BLAN-SIMI10-LS-100617-15-01, and LUNAPROBE under contract ANR-09-NANO-019-01 is acknowledged.

\section{References}

[1] Hövel H, Fritz S, Hilger A, Kreibig U and Vollmer M 1993 Width of cluster plasmon resonances: Bulk dielectric functions and chemical interface damping Phys. Rev. B 48 18178-88

[2] Yoffe A D 2001 Semiconductor quantum dots and related systems: Electronic, optical, luminescence and related properties of low dimensional systems Adv. Phys. 50 1-208

[3] Lombardi A, Grzelczak M P, Crut A, Maioli P, Pastoriza-Santos I, Liz-Marzán L M, Del Fatti N and Vallée F 2013 Optical Response of Individual Au-Ag@SiO2 Heterodimers ACS Nano 7 2522-31

[4] Michler P, Kiraz A, Becher C, Schoenfeld W V, Petroff P M, Zhang L, Hu E and Imamoglu A 2000 A quantum dot single-photon turnstile device. Science $2902282-5$

[5] Alivisatos P 2004 The use of nanocrystals in biological detection. Nat. Biotechnol. 22 47-52

[6] McFarland A D and Van Duyne R P 2003 Single Silver Nanoparticles as Real-Time Optical Sensors with Zeptomole Sensitivity Nano Lett. 3 1057-62 
[7] Stoll T, Maioli P, Crut A, Del Fatti N and Vallée F 2014 Advances in femto-nano-optics: ultrafast nonlinearity of metal nanoparticles Eur. Phys. J. B 87260

[8] Crut A, Maioli P, Del Fatti N and Vallée F 2014 Optical absorption and scattering spectroscopies of single nano-objects. Chem. Soc. Rev. 43 3921-56

[9] Andrade L H F, Laraoui A, Vomir M, Muller D, Stoquert J-P, Estournès C, Beaurepaire E and Bigot J-Y 2006 Damped Precession of the Magnetization Vector of Superparamagnetic Nanoparticles Excited by Femtosecond Optical Pulses Phys. Rev. Lett. 97127401

[10] Crut A, Maioli P, Del Fatti N and Vallée F 2014 Acoustic vibrations of metal nano-objects: Time-domain investigations Phys. Rep. 549 1-43

[11] Ambrose W P, Basché T and Moerner W E 1991 Detection and spectroscopy of single pentacene molecules in a p-terphenyl crystal by means of fluorescence excitation J. Chem. Phys. 95 7150-63

[12] Lichtman J W and Conchello J-A 2005 Fluorescence microscopy Nat. Methods 2910 - 919

[13] Kamada H, Gotoh H, Temmyo J, Takagahara T and Ando H 2001 Exciton Rabi Oscillation in a Single Quantum Dot Phys. Rev. Lett. 87246401

[14] Sönnichsen C, Franzl T, Wilk T, von Plessen G, Feldmann J, Wilson O and Mulvaney P 2002 Drastic reduction of plasmon damping in gold nanorods Phys. Rev. Lett. 88077402

[15] Liu M and Guyot-Sionnest P 2004 Synthesis and Optical Characterization of Au/Ag Core/Shell Nanorods J. Phys. Chem. B 108 5882-8

[16] Ignatovich F V. and Novotny L 2006 Real-Time and Background-Free Detection of Nanoscale Particles Phys. Rev. Lett. 96013901

[17] Arbouet A, Christofilos D, Del Fatti N, Vallée F, Huntzinger J, Arnaud L, Billaud P and Broyer M 2004 Direct measurement of the single-metal-cluster optical absorption Phys. Rev. Lett. 93 127401

[18] Christofilos D, Blancon J-C, Arvanitidis J, San Miguel A, Ayari A, Del Fatti N and Vallée F 2012 Optical Imaging and Absolute Absorption Cross Section Measurement of Individual Nanoobjects on Opaque Substrates: Single-Wall Carbon Nanotubes on Silicon J. Phys. Chem. Lett. 3 1176-81

[19] Dresselhaus M S, Dresselhaus G, Jorio A, Souza Filho A G and Saito R 2002 Raman spectroscopy on isolated single wall carbon nanotubes Carbon $\mathbf{4 0}$ 2043-61

[20] Dresselhaus M S, Dresselhaus G, Jorio A, Souza Filho A G, Pimenta M A and Saito R 2002 Single Nanotube Raman Spectroscopy Acc. Chem. Res. 35 1070-8

[21] Boyer D, Tamarat P, Maali A, Lounis B and Orrit M 2002 Photothermal imaging of nanometersized metal particles among scatterers. Science 297 1160-3

[22] Berciaud S, Cognet L, Blab G A and Lounis B 2004 Photothermal Heterodyne Imaging of Individual Nonfluorescent Nanoclusters and Nanocrystals Phys. Rev. Lett. 93257402 
[23] Baffou G, Bon P, Savatier J, Polleux J, Zhu M, Merlin M, Rigneault H and Monneret S 2012 Thermal Imaging of Nanostructures by Quantitative Optical Phase Analysis ACS Nano 6 24528

[24] Lo S S, Devadas M S, Major T A and Hartland G V. 2013 Optical detection of single nanoobjects by transient absorption microscopy Analyst 138 25-31

[25] Butet J, Duboisset J, Bachelier G, Russier-Antoine I, Benichou E, Jonin C and Brevet P-F 2010 Optical second harmonic generation of single metallic nanoparticles embedded in a homogeneous medium. Nano Lett. 10 1717-21

[26] Lippitz M, van Dijk M A and Orrit M 2005 Third-harmonic generation from single gold nanoparticles. Nano Lett. 5 799-802

[27] Masia F, Langbein W, Watson P and Borri P 2009 Resonant four-wave mixing of gold nanoparticles for three-dimensional cell microscopy. Opt. Lett. 34 1816-8

[28] Berciaud S, Cognet L, Poulin P, Weisman R B and Lounis B 2007 Absorption spectroscopy of individual single-walled carbon nanotubes. Nano Lett. 7 1203-7

[29] Beversluis M R, Bouhelier A and Novotny L 2003 Continuum generation from single gold nanostructures through near-field mediated intraband transitions Phys. Rev. B 68115433

[30] Berciaud S, Cognet L, Tamarat P and Lounis B 2005 Observation of intrinsic size effects in the optical response of individual gold nanoparticles. Nano Lett. 5 515-8

[31] Vietmeyer F, McDonald M P and Kuno M 2012 Single Nanowire Microscopy and Spectroscopy J. Phys. Chem. C 116 12379-96

[32] Blancon J-C, Paillet M, Tran H N, Than X T, Aberra Guebrou S, Ayari A, San Miguel A, Phan NM, Zahab A-A, Sauvajol J-L, Del Fatti N and Vallée F 2013 Direct measurement of the absolute absorption spectrum of individual semiconducting single-wall carbon nanotubes. Nat. Commun. 42542

[33] Celebrano M, Kukura P, Renn A and Sandoghdar V 2011 Single-molecule imaging by optical absorption Nat. Photonics 5 95-8

[34] Klar T, Perner M, Grosse S, von Plessen G, SpirkI W and Feldmann J 1998 Surface-plasmon resonances in single metallic nanoparticles Phys. Rev. Lett. 80 4249-52

[35] Krenn J R, Dereux A, Weeber J C, Bourillot E, Lacroute Y, Goudonnet J P, Schider G, Gotschy W, Leitner A, Aussenegg F R and Girard C 1999 Squeezing the Optical Near-Field Zone by Plasmon Coupling of Metallic Nanoparticles Phys. Rev. Lett. 82 2590-3

[36] Kreibig U and Vollmer M 1995 Optical Properties of Metal Clusters ed Springer Verlag (New York: Springer)

[37] Kelly K L, Coronado E, Zhao L L and Schatz G C 2003 The optical properties of metal nanoparticles: the influence of size, shape, and dielectric environment J. Phys. Chem. B 107 $668-77$ 
[38] Sagle L B, Ruvuna L K, Ruemmele J A and Van Duyne R P 2011 Advances in localized surface plasmon resonance spectroscopy biosensing. Nanomedicine 6 1447-62

[39] Tam F, Goodrich G P, Johnson B R and Halas N J 2007 Plasmonic enhancement of molecular fluorescence. Nano Lett. 7 496-501

[40] Sharma B, Frontiera R R, Henry A-I, Ringe E and Van Duyne R P 2012 SERS: Materials, applications, and the future Mater. Today 15 16-25

[41] Billaud P, Marhaba S, Cottancin E, Arnaud L, Bachelier G, Bonnet C, Del Fatti N, Lermé J, Vallée F, Vialle J-L, Broyer M and Pellarin M 2008 Correlation between the Extinction Spectrum of a Single Metal Nanoparticle and Its Electron Microscopy Image J. Phys. Chem. C 112 978-82

[42] Henry A-I, Bingham J M, Ringe E, Marks L D, Schatz G C and Van Duyne R P 2011 Correlated Structure and Optical Property Studies of Plasmonic Nanoparticles J. Phys. Chem. C 115 9291305

[43] Lermé J, Baida H, Bonnet C, Broyer M, Cottancin E, Crut A, Maioli P, Del Fatti N, Vallée F and Pellarin M 2010 Size dependence of the surface plasmon resonance damping in metal nanospheres J. Phys. Chem. Lett. 1 2922-8

[44] Baida H, Mongin D, Christofilos D, Bachelier G, Crut A, Maioli P, Del Fatti N and Vallée F 2011 Ultrafast nonlinear optical response of a single gold nanorod near its surface plasmon resonance Phys. Rev. Lett. 107057402

[45] Lombardi A, Grzelczak M P, Pertreux E, Crut A, Maioli P, Pastoriza-Santos I, Liz-Marzán L M, Vallée $F$ and Del Fatti N 2016 Fano Interference in the Optical Absorption of an Individual Gold-Silver Nanodimer. Nano Lett. 16 6311-6

[46] Vallée F and Del Fatti N 2013 Ultrafast Nonlinear Plasmonics Plasmonics in Metal Nanostructures: Theory and Applications ed T Shahbazyan and M Stockman (Springer Book Series)

[47] Bohren C F and Huffman D R 1983 Absorption and Scattering of Light by Small Particles (New York: Wiley)

[48] Van Dijk M A, Tchebotareva A L, Orrit M, Lippitz M, Berciaud S, Lasne D, Cognet L and Lounis B 2006 Absorption and scattering microscopy of single metal nanoparticles. Phys. Chem. Chem. Phys. 8 3486-95

[49] Lindfors K, Kalkbrenner T, Stoller P and Sandoghdar V 2004 Detection and Spectroscopy of Gold Nanoparticles Using Supercontinuum White Light Confocal Microscopy Phys. Rev. Lett. 93037401

[50] Hu M, Novo C, Funston A, Wang H, Staleva H, Zou S, Mulvaney P, Xia Y and Hartland G V. 2008 Dark-field microscopy studies of single metal nanoparticles: understanding the factors that influence the linewidth of the localized surface plasmon resonance. J. Mater. Chem. 18 194960 
[51] Sönnichsen C, Geier S, Hecker N E, von Plessen G, Feldmann J, Ditlbacher H, Lamprecht B, Krenn J R, Aussenegg F R, Chan V Z-H, Spatz J P and Möller M 2000 Spectroscopy of single metallic nanoparticles using total internal reflection microscopy Appl. Phys. Lett. 772949

[52] Thibodeaux C A, Kulkarni V, Chang W-S, Neumann O, Cao Y, Brinson B, Ayala-Orozco C, Chen C-W, Morosan E, Link S, Nordlander P and Halas N J 2014 Impurity-induced plasmon damping in individual cobalt-doped hollow Au nanoshells. J. Phys. Chem. B 118 14056-61

[53] Sönnichsen C, Franzl T, Wilk T, von Plessen G and Feldmann J 2002 Plasmon resonances in large noble-metal clusters New J. Phys. 493

[54] Mitra A, Deutsch B, Ignatovich F, Dykes C and Novotny L 2010 Nano-optofluidic detection of single viruses and nanoparticles. ACS Nano 4 1305-12

[55] Bingham J M, Willets K A, Shah N C, Andrews D Q and Van Duyne R P 2009 Localized Surface Plasmon Resonance Imaging? : Simultaneous Single Nanoparticle Spectroscopy and Diffusional Dynamics J. Phys. Chem. C 4 16839-42

[56] Knight M W, Fan J, Capasso F and Halas N J 2010 Influence of excitation and collection geometry on the dark field spectra of individual plasmonic nanostructures. Opt. Express $\mathbf{1 8}$ 2579-87

[57] Berciaud S, Cognet L and Lounis B 2008 Luminescence Decay and the Absorption Cross Section of Individual Single-Walled Carbon Nanotubes Phys. Rev. Lett. 101077402

[58] Gaiduk A, Yorulmaz M, Ruijgrok P V and Orrit M 2010 Room-temperature detection of a single molecule's absorption by photothermal contrast. Science 330 353-6

[59] Lasne D, Blab G A, Berciaud S, Heine M, Groc L, Choquet D, Cognet L and Lounis B 2006 Single nanoparticle photothermal tracking (SNaPT) of $5 \mathrm{~nm}$ gold beads in live cells. Biophys. J. 91 4598-604

[60] Berciaud S, Lasne D, Blab G A, Cognet L and Lounis B 2006 Photothermal heterodyne imaging of individual metallic nanoparticles: Theory versus experiment Phys. Rev. B 73045424

[61] Muskens O, Billaud P, Broyer M, Del Fatti N and Vallée F 2008 Optical extinction spectrum of a single metal nanoparticle: quantitative characterization of a particle and of its local environment Phys. Rev. B 78205410

[62] Lombardi A, Loumaigne M, Crut A, Maioli P, Del Fatti N, Vallée F, Spuch-Calvar M, Burgin J, Majimel J and Tréguer-Delapierre M 2012 Surface plasmon resonance properties of single elongated nano-objects: gold nanobipyramids and nanorods Langmuir 28 9027-33

[63] Muskens O L, Del Fatti N, Vallée F, Huntzinger J R, Billaud P and Broyer M 2006 Single metal nanoparticle absorption spectroscopy and optical characterization Appl. Phys. Lett. 88063109

[64] Billaud P, Marhaba S, Grillet N, Cottancin E, Bonnet C, Lermé J, Vialle J-L, Broyer M and Pellarin M 2010 Absolute optical extinction measurements of single nano-objects by spatial modulation spectroscopy using a white lamp. Rev. Sci. Instrum. 81043101 
[65] Muskens O L, Bachelier G, Del Fatti N, Vallée F, Brioude A, Jiang X and Pileni M-P 2008 Quantitative Absorption Spectroscopy of a Single Gold Nanorod J. Phys. Chem. C 112 8917-21

[66] Baida H, Billaud P, Marhaba S, Christofilos D, Cottancin E, Crut A, Lermé J, Maioli P, Pellarin M, Broyer M, Del Fatti N, Vallée F, Sánchez-Iglesias A, Pastoriza-Santos I and Liz-Marzán L M 2009 Quantitative determination of the size dependence of surface plasmon resonance damping in single Ag@SiO2 nanoparticles. Nano Lett. $93463-9$

[67] Baida H, Crut A, Maioli P, Nguyen T B, Nguyen D H, Del Fatti N and Vallée F 2011 Optical detection and femtosecond spectroscopy of a single nanoparticle Adv. Nat. Sci. Nanosci. Nanotechnol. 2035011

[68] Giblin J, Vietmeyer F, McDonald M P and Kuno M 2011 Single nanowire extinction spectroscopy. Nano Lett. 11 3307-11

[69] Davletshin Y R, Lombardi A, Cardinal M F, Juvé V, Crut A, Maioli P, Liz-Marzán L M, Vallée F, Del Fatti N and Kumaradas J C 2012 A Quantitative Study of the Environmental Effects on the Optical Response of Gold Nanorods ACS Nano 6 8183-93

[70] Ringe E, Langille M R, Sohn K, Zhang J, Huang J, Mirkin C A, Van Duyne R P and Marks L D 2012 Plasmon Length: A Universal Parameter to Describe Size Effects in Gold Nanoparticles J. Phys. Chem. Lett. 3 1479-83

[71] Blaber M G, Henry A-I, Bingham J M, Schatz G C and Van Duyne R P 2012 LSPR Imaging of Silver Triangular Nanoprisms?: Correlating Scattering with Structure Using Electrodynamics for Plasmon Lifetime Analysis J. Phys. Chem. C 116 393-403

[72] Munechika K, Smith J M, Chen Y and Ginger D S 2007 Plasmon Line Widths of Single Silver Nanoprisms as a Function of Particle Size and Plasmon Peak Position J. Phys. Chem. C 111 18906-11

[73] Kawabata A and Kubo R 1966 Electronic Properties of Fine Metallic Particles. II. Plasma Resonance Absorption J. Phys. Soc. Jpn. 21 1765-72

[74] Savage K J, Hawkeye M M, Esteban R, Borisov A G, Aizpurua J and Baumberg J J 2012 Revealing the quantum regime in tunnelling plasmonics. Nature 491 574-7

[75] Genzel L, Martin T P and Kreibig U 1975 Dielectric function and plasma resonances of small metal particles Z. Phys. B 21 339-46

[76] Pertreux E, Lombardi A, Florea I, Spuch-Calvar M, Gómez-Graña S, Ihiawakrim D, Hirlimann C, Ersen $\mathrm{O}$, Majimel J, Tréguer-Delapierre M, Hettich M, Maioli P, Crut A, Vallée F and Del Fatti N 2015 Surface Plasmon Resonance of an Individual Nano-object on an Absorbing Substrate: Quantitative Effects of Distance and 3D Orientation Adv. Opt. Mater.

[77] Vallée F 2007 Optical properties of metal nanoparticles Nanomaterials and Nanochemistry ed C Bréchignac, P Houdy and M Lahmani (Heidelberg: Springer Verlag) pp 197-228

[78] Winsemius P, Guerrisi M and Rosei R 1975 Splitting of the interband absorption edge in Au: Temperature dependence Phys. Rev. B 12 4570-2 
[79] Juvé V, Cardinal M F, Lombardi A, Crut A, Maioli P, Pérez-Juste J, Liz-Marzán L M, Del Fatti N and Vallée $F 2013$ Size-dependent surface plasmon resonance broadening in nonspherical nanoparticles: single gold nanorods Nano Lett. 13 2234-40

[80] Habteyes T G, Dhuey S, Wood E, Gargas D, Cabrini S, Schuck P J, Alivisatos A P and Leone S R 2012 Metallic adhesion layer induced plasmon damping and molecular linker as a nondamping alternative. ACS Nano 6 5702-9

[81] Liu M, Pelton M and Guyot-Sionnest P 2009 Reduced damping of surface plasmons at low temperatures Phys. Rev. B 79035418

[82] Wokaun A, Godon J P and Liao P F 1982 Radiation Damping in Surface-Enhanced Raman Scattering Phys. Rev. Lett. 48 957-60

[83] Grigorchuk N I 2012 Radiative damping of surface plasmon resonance in spheroidal metallic nanoparticle embedded in a dielectric medium J. Opt. Soc. Am. B 293404

[84] Novo C, Gomez D, Pérez-Juste J, Zhang Z, Petrova H, Reismann M, Mulvaney P and Hartland G V. 2006 Contributions from radiation damping and surface scattering to the linewidth of the longitudinal plasmon band of gold nanorods: a single particle study. Phys. Chem. Chem. Phys. $83540-6$

[85] Brown L V, Sobhani H, Lassiter J B, Nordlander P and Halas N J 2010 Heterodimers: plasmonic properties of mismatched nanoparticle pairs. ACS Nano 4 819-32

[86] Sonnefraud $Y$, Verellen N, Sobhani H, Vandenbosch G A E, Moshchalkov V V, Van Dorpe $P$, Nordlander P and Maier S A 2010 Experimental realization of subradiant, superradiant, and fano resonances in ring/disk plasmonic nanocavities. ACS Nano 4 1664-70

[87] Luk'yanchuk B, Zheludev N I, Maier S A, Halas N J, Nordlander P, Giessen H and Chong C T 2010 The Fano resonance in plasmonic nanostructures and metamaterials. Nat. Mater. 9 70715

[88] Zhang S, Bao K, Halas N J, Xu H and Nordlander P 2011 Substrate-induced Fano resonances of a plasmonic nanocube: a route to increased-sensitivity localized surface plasmon resonance sensors revealed. Nano Lett. 11 1657-63

[89] Coronado E A and Schatz G C 2003 Surface plasmon broadening for arbitrary shape nanoparticles: A geometrical probability approach J. Chem. Phys. 119 3926-34

[90] Kraus W A and Schatz G C 1983 Plasmon resonance broadening in small metal particles J. Chem. Phys. 79 6130-9

[91] Kreibig U 2008 Interface-induced dephasing of Mie plasmon polaritons Appl. Phys. B 93 79-89

[92] Almeida E, Moreira A C L, Brito-Silva A M, Galembeck A, de Melo C P, Menezes L de S and de Araújo C B 2012 Ultrafast dephasing of localized surface plasmons in colloidal silver nanoparticles: the influence of stabilizing agents Appl. Phys. B 108 9-16

[93] Wei J, Schaeffer N and Pileni M-P 2014 Ag nanocrystals: 1. Effect of ligands on plasmonic properties. J. Phys. Chem. B 118 14070-5 
[94] Kraus W A and Schatz G C 1983 Plasmon resonance broadening in spheroidal metal particles Chem. Phys. Lett. 99 353-7

[95] Kreibig U, Fragstein C v., Physik Z and March S R 1969 The limitation of electron mean free path in small silver particles Zeitschrift für Phys. 224 307-23

[96] Nehl C L, Grady N K, Goodrich G P, Tam F, Halas N J and Hafner J H 2004 Scattering Spectra of Single Gold Nanoshells Nano Lett. 4 2355-9

[97] Baida H, Christofilos D, Maioli P, Crut A, Del Fatti N and Vallée F 2011 Surface plasmon resonance spectroscopy of single surfactant-stabilized gold nanoparticles Eur. Phys. J. D 63 293-9

[98] Zijlstra P, Paulo P M R, Yu K, Xu Q-H and Orrit M 2012 Chemical interface damping in single gold nanorods and its near elimination by tip-specific functionalization. Angew. Chem. Int. Ed. $518352-5$

[99] Del Fatti N, Voisin C, Achermann M, Tzortzakis S, Christofilos D and Vallée F 2000 Nonequilibrium electron dynamics in noble metals Phys. Rev. B 61 16956-66

[100] Del Fatti N and Vallée F 2001 Ultrafast optical nonlinear properties of metal nanoparticles Appl. Phys. B 73 383-90

[101] Voisin C, Del Fatti N, Christofilos D and Vallée F 2001 Ultrafast electron dynamics and optical nonlinearities in metal nanoparticles J. Phys. Chem. B 105 2264-80

[102] Juvé V, Scardamaglia M, Maioli P, Crut A, Merabia S, Joly L, Del Fatti N and Vallée F 2009 Cooling dynamics and thermal interface resistance of glass-embedded metal nanoparticles Phys. Rev. B 80195406

[103] Stoll T, Maioli P, Crut A, Rodal-Cedeira S, Pastoriza-Santos I, Vallée F and Del Fatti N 2015 Time-Resolved Investigations of the Cooling Dynamics of Metal Nanoparticles: Impact of Environment J. Phys. Chem. C 119 12757-64

[104] Hartland G V. 2011 Optical studies of dynamics in noble metal nanostructures Chem. Rev. 111 3858-87

[105] Rosei R 1974 Temperature modulation of the optical transitions involving the Fermi surface in Ag: Theory Phys. Rev. B 10 474-83

[106] Voisin C, Christofilos D, Del Fatti N, Vallée F, Prével B, Cottancin E, Lermé J, Pellarin M and Broyer M 2000 Size-dependent electron-electron interactions in metal nanoparticles Phys. Rev. Lett. 85 2200-3

[107] Arbouet A, Voisin C, Christofilos D, Langot P, Del Fatti N, Vallée F, Lermé J, Celep G, Cottancin E, Gaudry M, Pellarin M, Broyer M, Maillard M, Pileni M-P and Treguer M 2003 Electronphonon scattering in metal clusters Phys. Rev. Lett. 90177401

[108] Gurzhi R N 1959 Mutual Electron Correlations in Metal Optics Sov. Phys. JETP 8 673-5 
[109] Parkins G R, Lawrence W E and Christy R W 1981 Intraband optical conductivity $\sigma(\omega, T)$ of $\mathrm{Cu}, \mathrm{Ag}$, and Au: Contribution from electron-electron scattering Phys. Rev. B 23 6408-16

[110] Smith J B and Ehrenreich H 1982 Frequency dependence of the optical relaxation time in metals Phys. Rev. B 25 923-30

[111] Zavelani-Rossi M, Polli D, Kochtcheev S, Baudrion A-L, Béal J, Kumar V, Molotokaite E, Marangoni M, Longhi S, Cerullo G, Adam P-M and Della Valle G 2015 Transient Optical Response of a Single Gold Nanoantenna: The Role of Plasmon Detuning ACS Photonics 2 5219

[112] Masia F, Langbein W and Borri P 2012 Measurement of the dynamics of plasmons inside individual gold nanoparticles using a femtosecond phase-resolved microscope Phys. Rev. B 85 235403

[113] Masia F, Langbein W and Borri P 2013 Polarization-resolved ultrafast dynamics of the complex polarizability in single gold nanoparticles. Phys. Chem. Chem. Phys. 15 4226-32

[114] Johnson P B and Christy R W 1972 Optical Constants of the Noble Metals Phys. Rev. B 6 43709

[115] Olmon R L, Slovick B, Johnson T W, Shelton D, Oh S-H, Boreman G D and Raschke M B 2012 Optical dielectric function of gold Phys. Rev. B 86235147

[116] Miroshnichenko A E, Flach S and Kivshar Y S 2010 Fano resonances in nanoscale structures Rev. Mod. Phys. 822257

[117] Halas N J, Lal S, Chang W-S, Link S and Nordlander P 2011 Plasmons in strongly coupled metallic nanostructures. Chem. Rev. 111 3913-61

[118] Bachelier G, Russier-Antoine I, Benichou E, Jonin C, Del Fatti N, Vallée F and Brevet P-F 2008 Fano Profiles Induced by Near-Field Coupling in Heterogeneous Dimers of Gold and Silver Nanoparticles Phys. Rev. Lett. 101197401

[119] Baida H, Christofilos D, Maioli P, Crut A, Del Fatti N and Vallée F 2008 Surface plasmon resonance linear and nonlinear response in a single nanorod Proc. SPIE 7033, Plasmonics: Nanoimaging, Nanofabrication, and Their Applications IV ed S Kawata, V M Shalaev and D P Tsai p 703319

[120] Guerrisi M, Rosei R and Winsemius P 1975 Splitting of the interband absorption edge in Au Phys. Rev. B 12 557-63

[121] Palik E D 1985 Handbook of Optical Constants of Solids vol 1 (Orlando: Academic Press) 

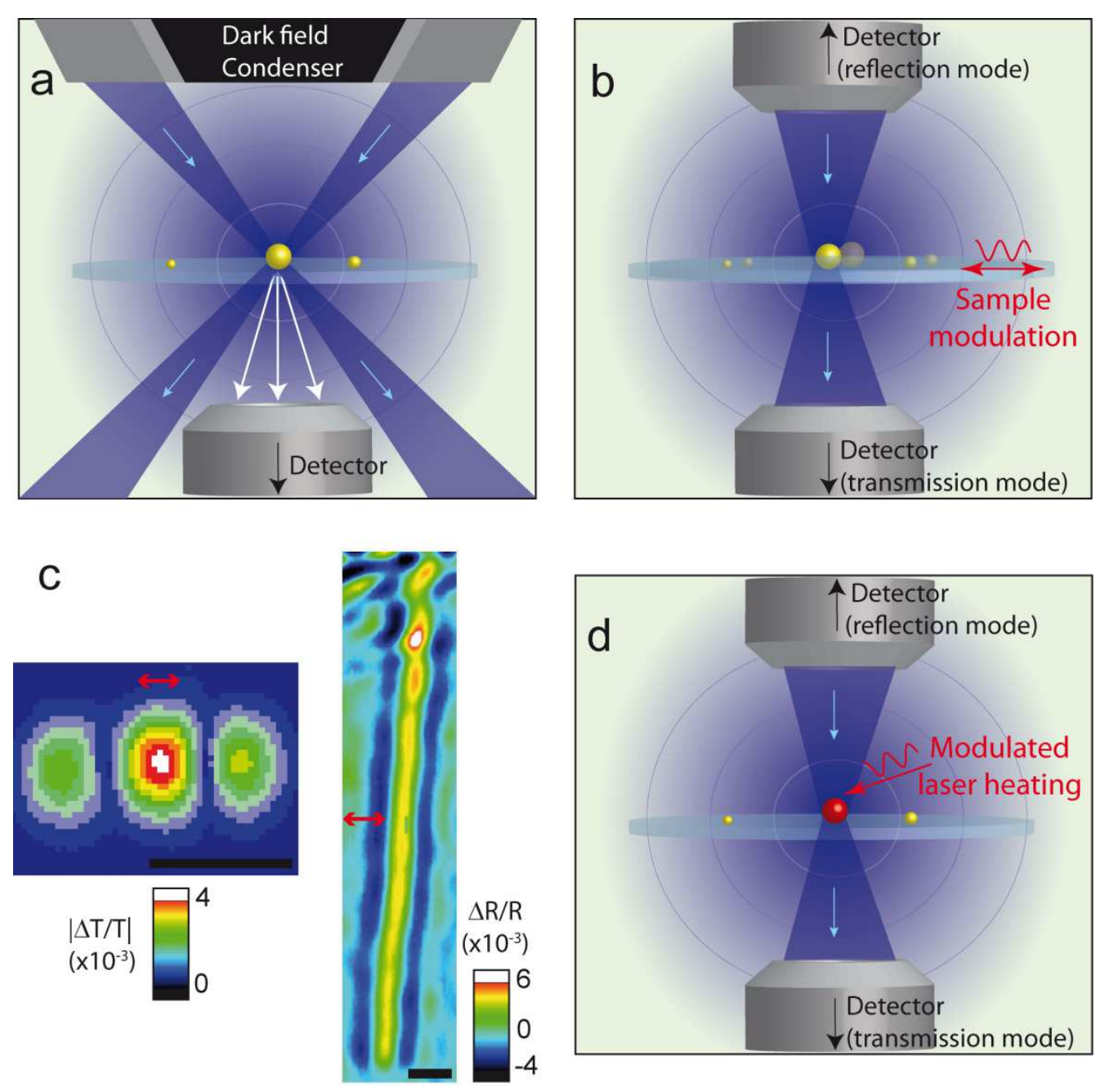

Figure 1. Most commonly used single-particle spectroscopy approaches. The incident light beam is represented in dark blue (with arrows showing its propagation direction). The wave scattered by an illuminated nano-object is shown in lighter blue. a) Dark-field microscopy. Illumination is achieved using oblique rays. Only forward scattered rays collected by the objective reach the detector. b) Spatial modulation spectroscopy. It combines diffractionlimited illumination of a single isolated nano-object and sample position modulation. Detection can be performed either in transmission (bottom objective) or in reflection (top objective). c) Examples of images in a spatial modulation microscope. Left: T-SMS image of a single gold nanorod $(\approx 30 \mathrm{~nm}$ length, $\approx 10 \mathrm{~nm}$ diameter) obtained at $750 \mathrm{~nm}$. Right: R-SMS image of a single carbon nanotube of $1.6 \mathrm{~nm}$ diameter at $675 \mathrm{~nm}$. Sample modulation direction is shown by red arrows. Scale bars: $1 \mu \mathrm{m}$. d) Photothermal imaging. A first laser beam periodically heats up an absorbing nano-object and its environment. This results in a modulation of the transmission/reflection of a second laser beam. 


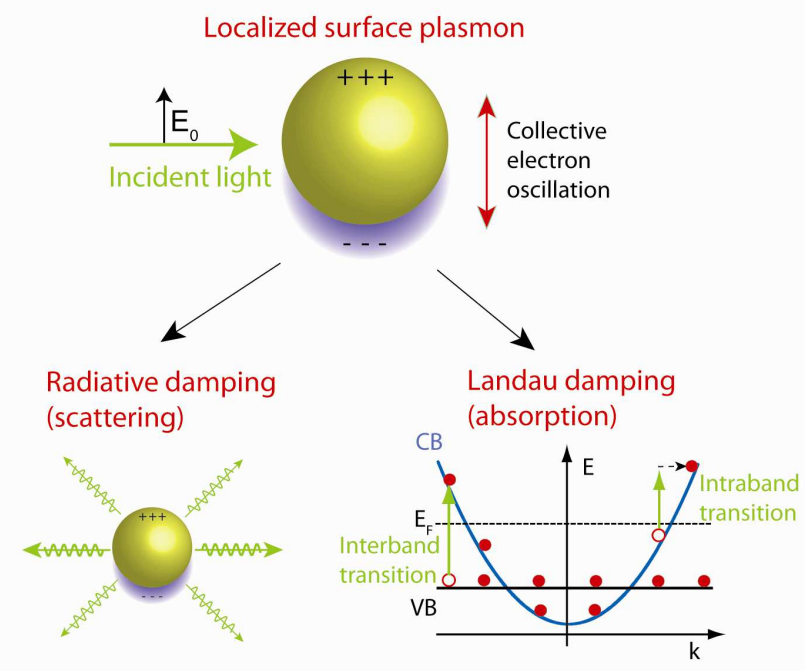

b
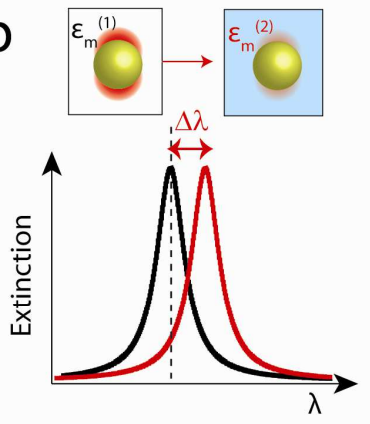

C
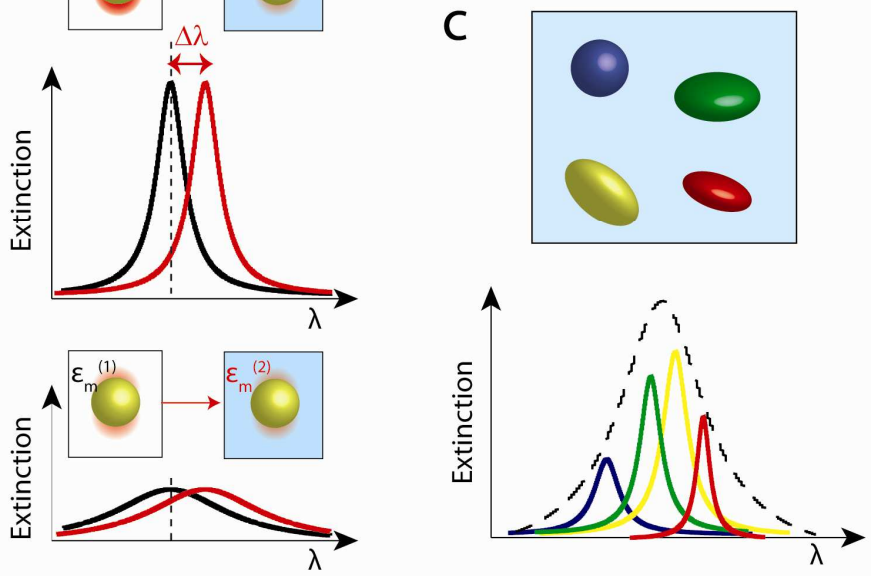

Figure 2. Localized surface plasmon resonance of metal nanoparticles. a) Localized surface plasmons are collective electron oscillations excited by an incident light beam. They decay through both radiative (Rayleigh scattering of the incident light) and non-radiative (Landau damping, i.e., single electron excitations) processes. In the latter case, an electron can be promoted to an unoccupied state (i.e., above Fermi energy $\mathrm{E}_{\mathrm{F}}$ in the conduction band $\mathrm{CB}$, involving sp electronic states in noble metals) by either interband (implying an initial state in the valence band $\mathrm{VB}$, involving $\mathrm{d}$ states in noble metals) or intraband (initial state in the $\mathrm{CB}$ ) transitions. b) SPR-based biosensing: principle. The SPR properties of a nanoparticle in solution are sensitive to the dielectric properties of its environment $\left(\varepsilon_{m}\right)$. SPR is thus displaced ( $\Delta \lambda$ wavelength shift) by solvent change or molecular binding at the nanoparticle surface. For a given $\Delta \lambda$, the differential signal at the measurement wavelength (dashed vertical line) increases with the resonance quality factor, enabling more sensitive detection. 
c) Advantage of single particle over ensemble experiments, which suffer from interparticle dispersion in size, shape and orientation (schematic drawing). For metal nanoparticles, this notably translates into shifts of their SPR positions. Ensemble spectra are therefore typically much broader than single-particle ones and hardly allow quantitative analysis of the surface plasmon damping processes shown in a). 

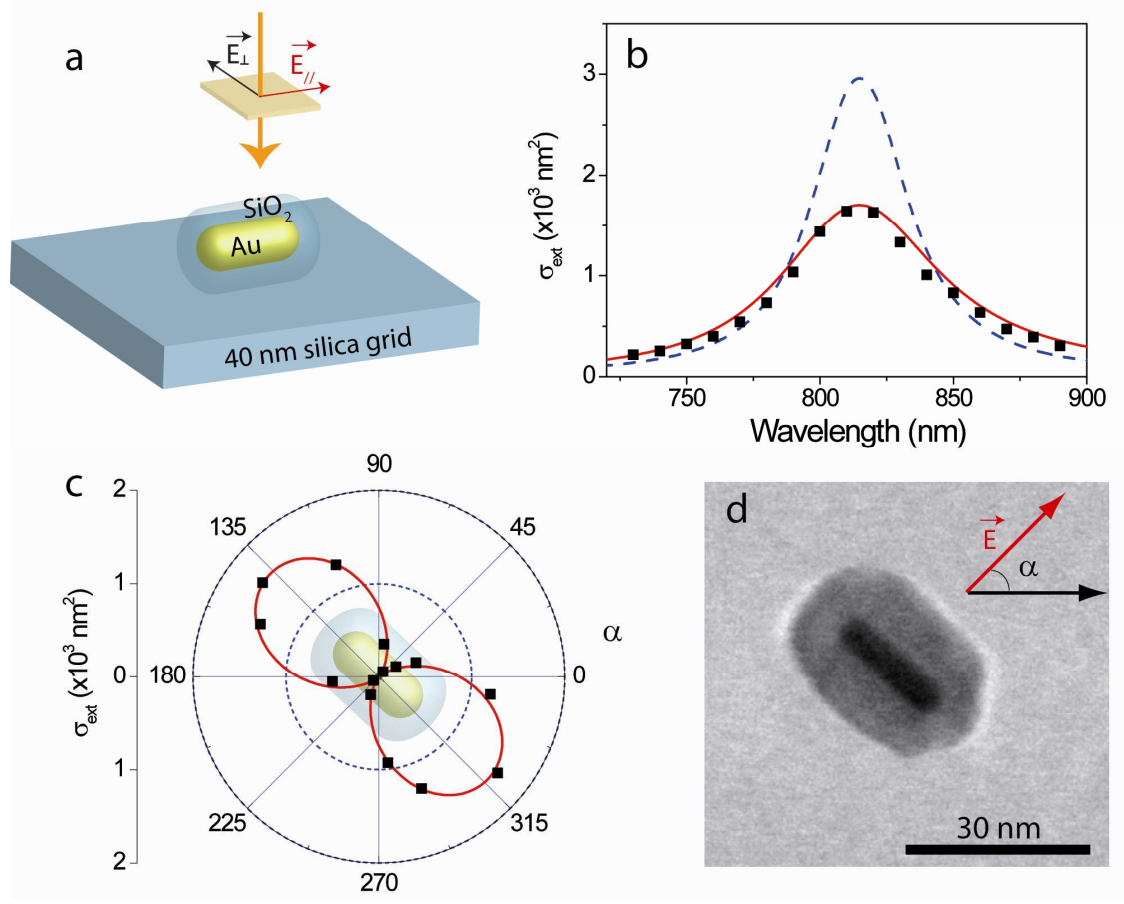

Figure 3. SMS quantitative linear spectroscopy of a single nanoparticle. a) Experimental geometry for investigating a single $\mathrm{Au} @ \mathrm{SiO}_{2}$ nanorod deposited on a silica substrate with linearly polarized light. b) Black squares: absolute extinction spectrum measured by SMS for light polarized along the nanorod large axis $\left(E_{/ /}\right.$in a)), corresponding to the longitudinal SPR response of the nanorod. Dashed blue line: extinction spectrum computed by finite-element modeling, using the gold bulk dielectric constants. Nanorod length and diameter were adjusted to reproduce the experimental SPR position and area in the energy domain (optimal fitting values are $L=33.4 \mathrm{~nm}$ and $D=8.8 \mathrm{~nm}$ ). An excellent reproduction of the experimental spectrum is obtained with a Lorentzian profile (red solid line) with the same spectral position and area, but an increased SPR width (corresponding to setting $\gamma_{s}=60 \mathrm{meV}$, see equation 8). c) Black squares: polarization dependence of the nanorod extinction cross section at $810 \mathrm{~nm}\left(\alpha=0^{\circ}\right.$ corresponds to light polarized horizontally in the sample plane). Red

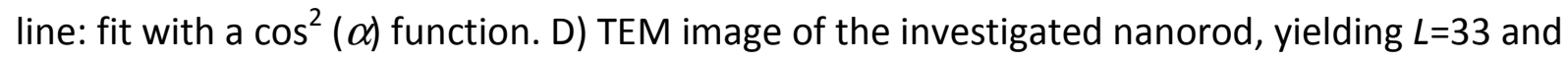
$D=8.8 \mathrm{~nm}$. The long axis orientation corresponds to the polarization direction maximizing extinction in c). 


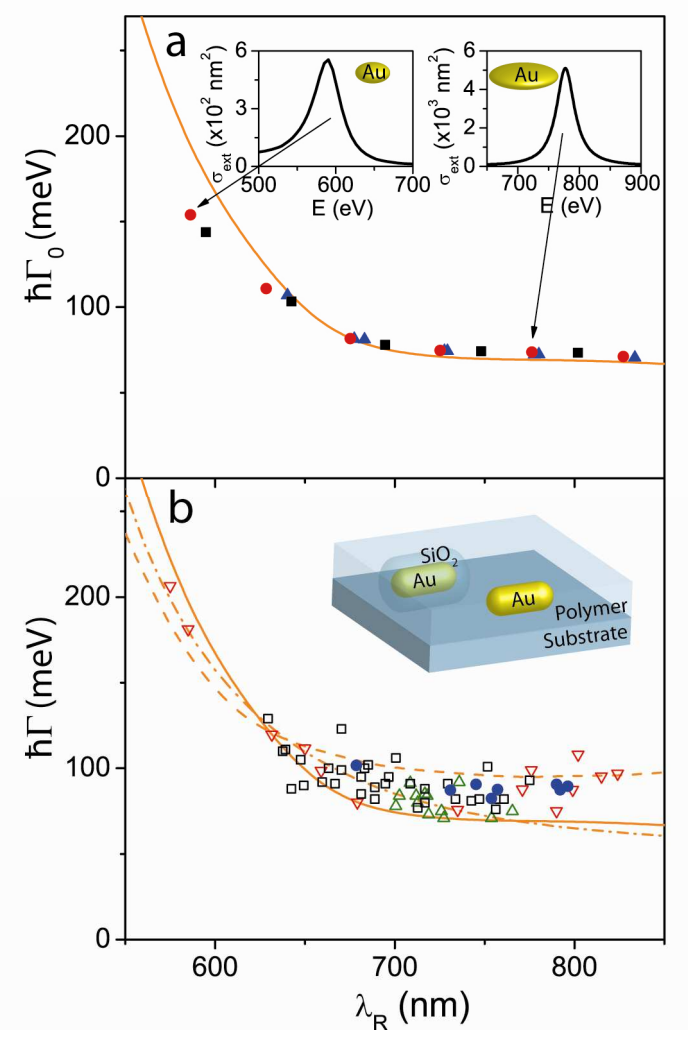

Figure 4. SPR width of individual elongated gold nano-objects. a) $\Gamma_{0}$ values computed using equation 7 (solid line), and from a Lorentzian fit of the numerically computed extinction cross section for nanoellipsoids (red circles), nanorods (black squares) and nanobipyramids (blue triangles) of small size (diameter $D=10 \mathrm{~nm}$, variable aspect ratios from 2 to 4.5). Gold dielectric function was taken from Johnson and Christy [114] and a uniform environment with 1.45 refractive index was considered. At small wavelengths, $\Gamma_{0}$ increases due to interband transitions, and SPR becomes increasingly asymmetric (left and right insets present the SPR spectra of nanoellipsoids with aspect ratios of 2 and 4, respectively), so that the widths extracted from Lorentzian fits of numerical spectra are less reliable. b) SPR width measured on Au nanorods (black squares [84], green triangles [15], red triangles [65,119] and blue circles [79]), embedded in a polymer layer providing them with an optically quasiuniform environment with $\approx 1.45$ refractive index (insert). Nanorod sizes between $13 \mathrm{~nm}<D$ $<20 \mathrm{~nm}$ and $40 \mathrm{~nm}<L<60 \mathrm{~nm}$ are estimated from mean sample dimensions [15,65,84,119] or measured at single particle level by optical size determination [79]. Lines are the $\Gamma_{0}$ values computed with equation 7 using gold dielectric function from Johnson and Christy [114] (solid line, same as in a), Guerrisi et al. [120] (dashed line) and Olmon et al. [115] (dashdotted line). 

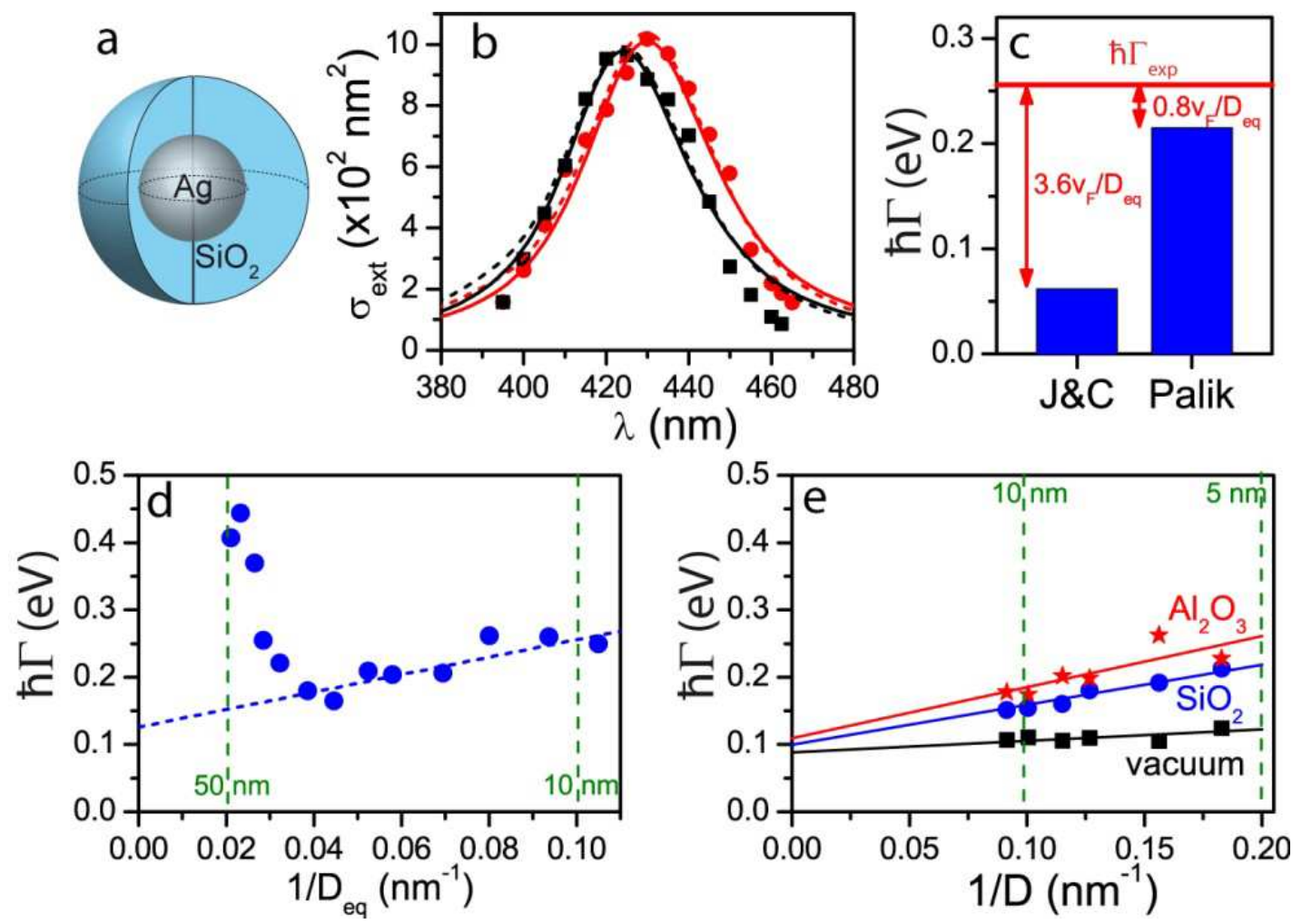

Figure 5. SPR width of individual $\mathrm{Ag} @ \mathrm{SiO}_{2}$ nanospheres. a) Schematic morphology of an $\mathrm{Ag} @ \mathrm{SiO}_{2}$ nanosphere. b) $\sigma_{\text {ext }}$ spectra measured by SMS for a single $\mathrm{Ag} @ \mathrm{SiO}_{2}$ nanoparticle for two orthogonal directions of the linear light polarization (black squares and red circles) corresponding to extrema of $\sigma_{\text {ext }}$ at a given wavelength. Full and dashed lines are fits using Johnson and Christy [114] and Palik [121] bulk silver dielectric functions corrected for size effects. The Ag nanoparticle was assumed of prolate shape with its equivalent diameter, aspect ratio $\eta$ and $\gamma_{s}$ (or, equivalently, $g_{s}$ using equation 9) as free parameters. Optimal fitting parameters are $D_{e q}=14.4(14.9) \mathrm{nm}, \eta=1.03$ (1.03) and $g_{s}=1.8(0.4)$ for Johnson and Christy (Palik) tables. c) Deduced bulk-like and radiative $\left(\Gamma_{0}+\Gamma_{r}\right)$ and surface $\left(\gamma_{s}\right)$ contributions to experimental SPR width $\Gamma_{\text {exp }}$. This evidences the large dependence of $\gamma_{s}\left(g_{s}\right)$ on the used table and precludes its estimation from a direct fit (see main text). d) Experimental size dependence of SPR width on individual optically determined nanoparticle size [66]. Each data point corresponds to a single nanoparticle measurement. The dashed blue line is a linear fit of the data with $\Gamma_{0}=125 \mathrm{meV}$ and $g_{s}=0.7$ (equations 8 and 9). e) SPR width computed using a quantum model for a silver nanoparticle embedded in vacuum (black triangles), silica (blue circles) and alumina (red stars) [43]. Lines correspond to linear fits of these computations. Adapted with permission from references [43,66]. Copyright 2009 and 2010 American Chemical Society. 

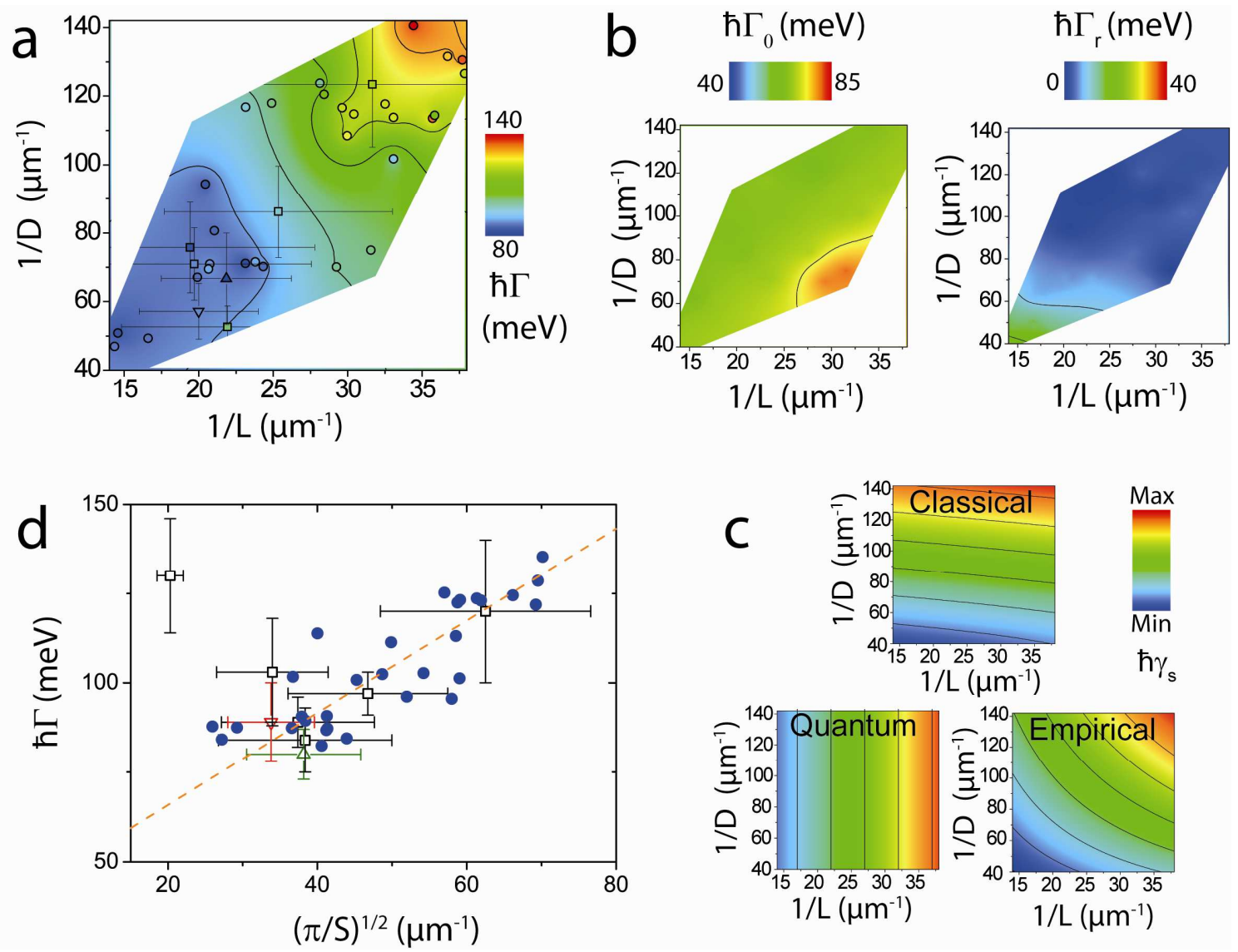

Figure 6. SPR width of individual Au nanorods. a) SPR width of individual Au@SiO nanorods $_{2}$ measured by SMS (circles) and deduced 2D color plot. Nanorod dimensions (length $L$ and diameter $D$ ) were optically determined [79]. Data points with error bars correspond to previous studies on Au rods $[15,65,84,119]$ (same symbols as in Figure 4) without optical size determination, the bars showing the dispersion of the nanorod solutions. b) Computed 2D plot of $\Gamma_{0}$ (using equation 7) and $\Gamma_{r}$ (using finite-element modeling) with Johnson and Christy table. c) Expected 2D plots of $\gamma_{s}$ in the context of classical billiard models (top), simple quantum models (bottom left), and using the empirical relation $\gamma_{s} \propto(\pi / S)^{1 / 2}$ (bottom right). d) Measured SPR width (see a)) plotted as a function of $(\pi / S)^{1 / 2}$ (same symbols as in Figure 4). The dashed orange line is a linear fit with $\Gamma_{0}=40 \mathrm{meV}$ and $A=1.3$ (see equation 10). Adapted with permission from reference [79]. Copyright 2013 American Chemical Society. 

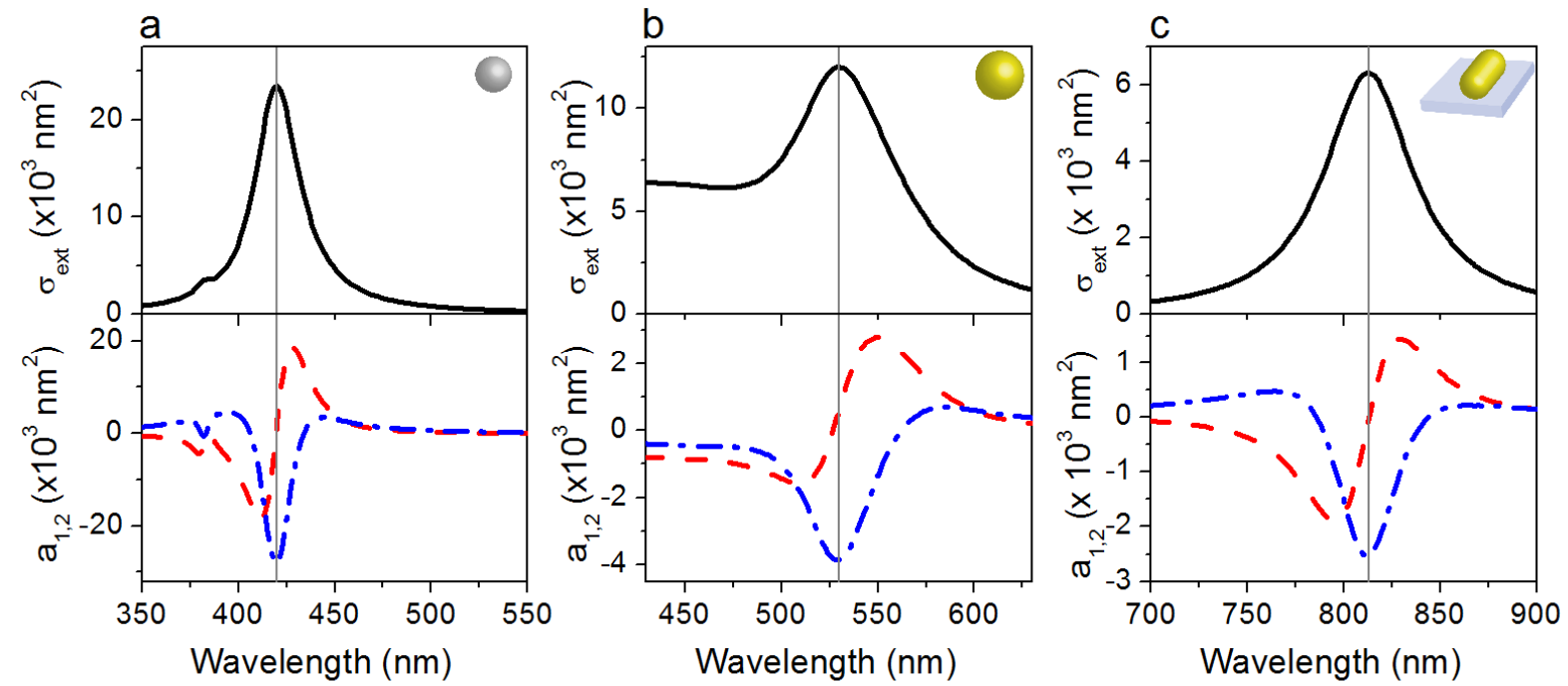

Figure 7. Computed extinction cross sections $\sigma_{\text {ext }}$ and derivatives $a_{1,2}$ for model metal nanoobjects. a) $\sigma_{\text {ext }}$ of $\mathrm{D}=40 \mathrm{~nm}$ silver nanosphere calculated by multipolar Mie theory with $g_{s}=0.7$, environment refractive index $\mathrm{n}=1.48$ and Johnson \& Christy bulk dielectric functions (upper panel, solid line). Its $a_{1}$ and $a_{2}$ derivatives (equation 11 ) are computed by numerical derivation of the linear extinction spectrum relatively to $\varepsilon_{1}$ and $\varepsilon_{2}$ (lower panel, red dashed and blue dash-dotted lines, respectively). Vertical gray bars indicate the SPR position (420 $\mathrm{nm}$ ). b) Same as a) for $\mathrm{D}=60 \mathrm{~nm}$ gold nanospheres with $\mathrm{n}=1.28$ (SPR at $530 \mathrm{~nm}$ ). c) Extinction cross section of a $43 \times 12 \mathrm{~nm}$ gold nanorod deposited on a glass substrate (SPR at $810 \mathrm{~nm}$ ) computed by Finite Element Modeling [44]. 


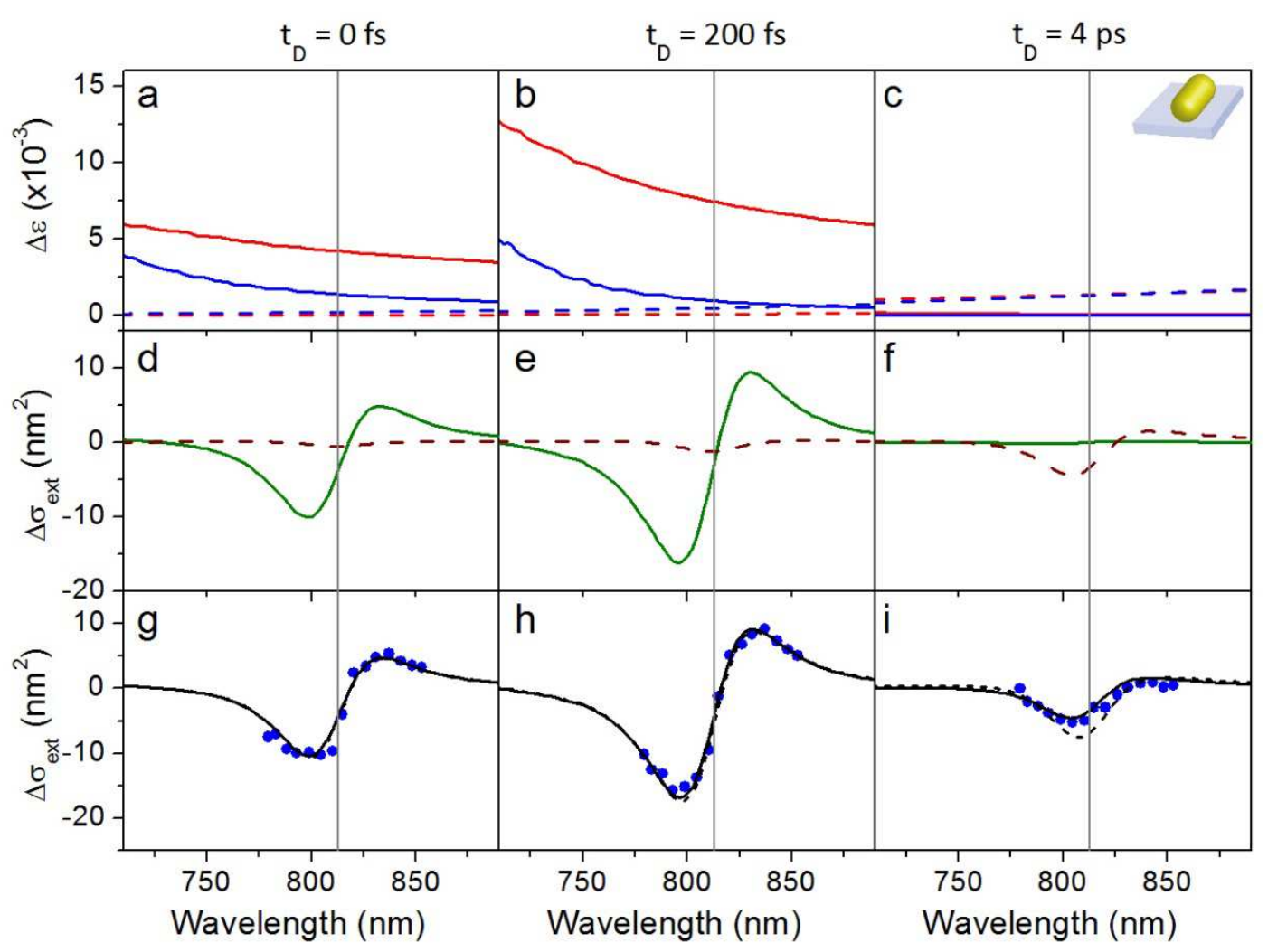

Figure 8. Ultrafast optical response of a gold nanorod after femtosecond excitation. a-c) Computed dielectric functions modifications $\Delta \varepsilon$ after excitation of the nanorod of Figure 7c by $130 \mathrm{fs}$ pump pulse at $400 \mathrm{~nm}$ for delays $t_{D}$ corresponding to $0 \mathrm{fs}, 200 \mathrm{fs}$ and 4 ps. $t_{D}=0$ is defined as the time at which half of the pump pulse energy has been transferred to the nano-object. The excitation corresponds to an electronic initial equivalent temperature increase $\Delta T_{\text {exc }}=125 \mathrm{~K}$. Equivalent electron and lattice temperature changes are $\Delta T_{e}=56,98$, $6 \mathrm{~K}$ and $\Delta T_{L}=0.004,0.07,0.9 \mathrm{~K}$ for the three delays. Solid (dashed) lines correspond to interband contribution $\Delta \varepsilon^{i b}$ (Drude contribution $\Delta \varepsilon^{D}$ ) to the real $\left(\Delta \varepsilon_{1}\right.$, red) and imaginary $\left(\Delta \varepsilon_{2}\right.$, blue) parts of the metal dielectric function. $d$-f) Computed $\Delta \sigma_{\text {ext }}$ interband (solid line) and Drude (dashed line) contributions (equation 11). g-i) Experimental (dots) and computed (lines) $\Delta \sigma_{\text {ext }}$ including both interband and Drude contributions (with $\hbar \gamma_{0}=20 \mathrm{meV}$, solid line, or $45 \mathrm{meV}$, dashed line) to the extinction cross section modifications. 

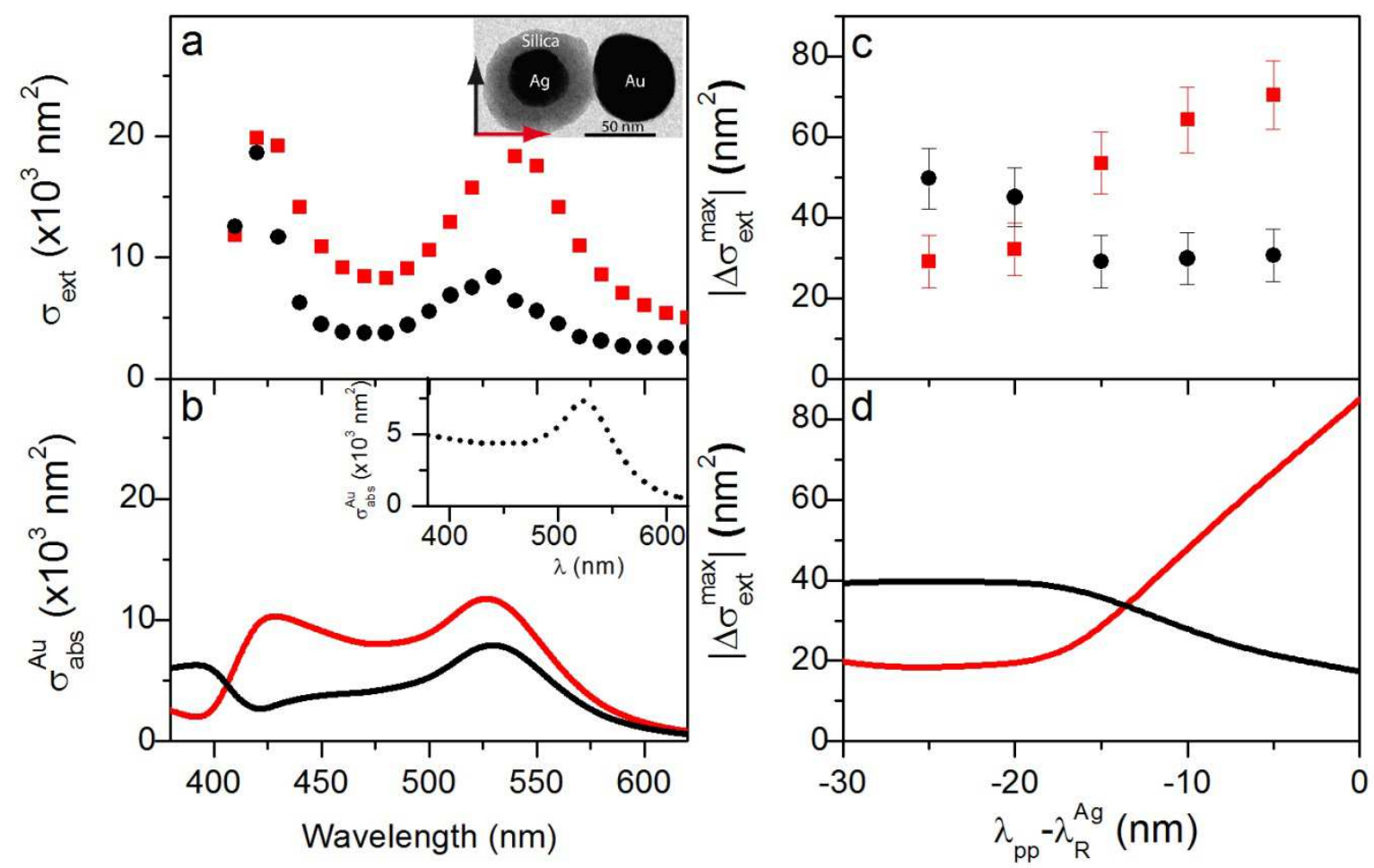

Figure 9. Fano effect in the absorption of an individual $\mathrm{Ag} @ \mathrm{SiO}_{2}-\mathrm{Au}$ nanodimer. Red (black) lines and symbols correspond to light polarization parallel (orthogonal) to the dimer axis. a) Extinction cross section of an individual $\mathrm{Ag} @ \mathrm{SiO}_{2}-\mathrm{Au}$ nanodimer deposited on silica substrate measured by SMS [45]. Inset: TEM image of the dimer (Au and Ag particle mean diameters $D_{\mathrm{Au}}=58 \mathrm{~nm}$ and $D_{\mathrm{Ag}}=40 \mathrm{~nm}$, and silica shell thickness $t=16 \mathrm{~nm}$ ). b) Computed absorption cross section of the Au component of the dimer, $\sigma_{\mathrm{abs}}^{\mathrm{Au}}$, under the influence of the nearby silver nanoparticle [45]. The spectral features and polarization dependence of gold absorption within the dimer at $\approx 420 \mathrm{~nm}$ are consequence of Fano interference. Inset: polarization independent absorption cross-section of an isolated Au nanoparticle. c) Maximal amplitudes of the dimer extinction cross-section change measured at $\lambda_{\mathrm{pr}}=530 \mathrm{~nm}$ as a function of pump polarization and wavelength relative to the Ag-like SPR position $\lambda_{R}^{A g}\left(\lambda_{\mathrm{pp}}\right.$ from $400 \mathrm{~nm}$ to $420 \mathrm{~nm}$, pump fluence $F_{\mathrm{pp}}=1.2 \mu \mathrm{J} / \mathrm{cm}^{2}$ ). d) Maximal amplitudes of the dimer extinction cross-section change computed at $\lambda_{\mathrm{pr}}=530 \mathrm{~nm}$ as a function of pump polarization and wavelength for the same excitation conditions as in experiments, assuming the gold absorption cross section plotted in b). Adapted with permission from reference [45]. Copyright 2013 American Chemical Society. 